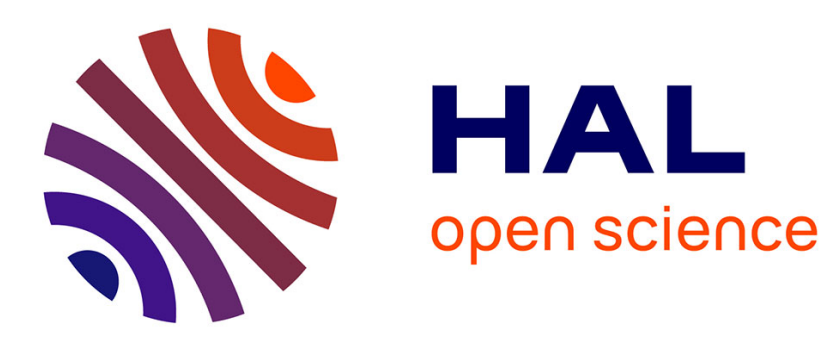

\title{
Diffusive representations for the analysis and simulation of flared acoustic pipes with visco-thermal losses
}

Thomas Hélie, Denis Matignon

\section{To cite this version:}

Thomas Hélie, Denis Matignon. Diffusive representations for the analysis and simulation of flared acoustic pipes with visco-thermal losses. Mathematical Models and Methods in Applied Sciences, 2006, 16 (4), pp.503-536. 10.1142/S0218202506001248 . hal-01373407

\section{HAL Id: hal-01373407 \\ https://hal.science/hal-01373407}

Submitted on 28 Sep 2016

HAL is a multi-disciplinary open access archive for the deposit and dissemination of scientific research documents, whether they are published or not. The documents may come from teaching and research institutions in France or abroad, or from public or private research centers.
L'archive ouverte pluridisciplinaire HAL, est destinée au dépôt et à la diffusion de documents scientifiques de niveau recherche, publiés ou non, émanant des établissements d'enseignement et de recherche français ou étrangers, des laboratoires publics ou privés. 


\section{Open Archive TOULOUSE Archive Ouverte (OATAO)}

OATAO is an open access repository that collects the work of Toulouse researchers and makes it freely available over the web where possible.

This is an author-deposited version published in: http://oatao.univ-toulouse.fr/ Eprints ID: 16165

To link to this article: DOI: $10.1142 / \mathrm{S} 0218202506001248$

URL: http://dx.doi.org/10.1142/S0218202506001248

To cite this version: Hélie, Thomas and Matignon, Denis Diffusive representations for the analysis and simulation of flared acoustic pipes with visco-thermal losses. (2006) Mathematical Models and Methods in Applied Sciences, vol. 16 ( $\left.\mathrm{n}^{\circ} 4\right)$. pp. 503-536. ISSN 0218-2025

Any correspondence concerning this service should be sent to the repository administrator: staff-oatao@listes-diff.inp-toulouse.fr 


\title{
DIFFUSIVE REPRESENTATIONS FOR THE ANALYSIS AND SIMULATION OF FLARED ACOUSTIC PIPES WITH VISCO-THERMAL LOSSES
}

\author{
TH. HÉLIE \\ Laboratoire des Sciences et Technologies de la Musique et du Son, \\ CNRS UMR 9912, Équipe Analyse/Synthése - Ircam, \\ Centre Georges Pompidou, 1, place Igor Stravinsky, \\ F-75004 Paris, France \\ thomas.helie@ircam.fr \\ D. MATIGNON \\ Dépt. Traitement du Signal et des Images, \\ Télécom Paris, CNRS UMR 5141, \\ 37-39, rue Dareau, F-75014 Paris, France \\ matignon@tsi.enst.fr
}

Communicated by M. Fabrizio

\begin{abstract}
Acoustic waves travelling in axisymmetric pipes with visco-thermal losses at the wall obey a Webster-Lokshin model. Their simulation may be achieved by concatenating scattering matrices of elementary transfer functions associated with nearly constant parameters (e.g. curvature). These functions are computed analytically and involve diffusive pseudo-differential operators, for which we have representation formula and inputoutput realizations, yielding direct numerical approximations of finite order. The method is based on some involved complex analysis.

Keywords: Wave propagation; pseudo-differential operators; complex analysis of multivalued functions; diffusive representations.
\end{abstract}

\section{Introduction}

In the context of real-time simulation of virtual musical intruments, a model of acoustic propagation in axisymmetric air-filled pipes is considered. This model, which depends on a single space variable, takes two phenomena into account. The curvature of the pipe is described by a Webster equation. The visco-thermal losses induced by the wall are described by a Lokshin equation with a fractional time-derivative of order one-half. The Webster-Lokshin model can be recast in the framework of coupled hyperbolic-parabolic partial differential equations. 
The present work is aimed at obtaining an input-output system which is modular (connecting pipes amounts to connecting systems) and which makes a lowcost simulation in the time domain possible. Such a formulation does not involve the computation of the acoustic state over the whole guide as standard methods of numerical analysis of PDEs do, e.g. finite differences or finite elements, but only at the boundaries. This can noticeably reduce the cost of computation due to the weight of the fractional integro-differential operators: these operators must be computed at each node of the mesh in standard methods, whereas they are embedded in the input-ouput system in the other case. Nevertheless, standard methods are currently studied and should provide a reference for making comparisons. ${ }^{1,2}$

We propose to describe the solutions of the Webster-Lokshin model by a forward-backward wave decomposition. The definition of these waves extends the exact decoupled cases of planar waves in lossless cylinders and spherical waves in lossless cones. To our best knowledge, this definition is new. The governing equations of these waves are two coupled partial differential equations of first order both in time and space. Taking their causal Laplace transform with respect to time leads to two coupled ordinary differential equations in space. If the curvature and loss coefficients are constant with respect to space, the scattering matrix can be derived analytically. It is proven to correspond to a causal system.

In this input-output approach, we consider transfer functions, as Laplace transforms of causal functions. The interest in the quite involved complex analysis of these transfer functions is twofold. On the one hand, this analysis helps and enhances understanding of the mathematical structure and hence the physical phenomena and properties. Among them, pure delays and pure diffusion kernels are exhibited, wavetrain decomposition is performed, long-time asymptotics are derived. On the other hand, this analysis is a means which reveals the necessity for the stable approximation of these transfer functions as finite order differential equations with delays. It yields an efficient tool for numerical simulation of the input-output system.

In the case of non-negative curvatures, the representation and approximation technique is based on the so-called diffusive representations. The fundamental solution of pseudo-differential operator of diffusive type can be decomposed on a continuous family of purely damped exponentials. A straightforward extension is required here, which makes use of a decomposition on a continuous family of damped oscillating exponentials. An approximation of such a representation is then performed by picking up a finite subset of this family. Now, as exponentials are solutions of first-order ordinary differential equations, the input/output relation based on the diffusive representations can be easily implemented with a set of such equations. This yields an input/state/output relation, also called a diffusive realization in the sense of systems theory (the Hilbert state space is of infinite dimension in the exact case and of finite dimension in the approximated case); the stability properties are guaranteed. A straightforward implementation makes a causal computation possible in the time domain, the computational cost being much lower than that 
of a convolution with a truncated impulse response. This feature is all the more interesting than the convolution kernels involved by visco-thermal losses and the curvature have slow decays, which forbid short-time truncation.

This paper is organized as follows. Section 2 introduces the Webster-Lokshin model. Locally travelling waves are defined. The corresponding adimensional problem is formulated in the Laplace domain. The scattering matrix is derived. The case of a baffled bell is detailed. Section 3 introduces the mathematical framework of diffusive representations of pseudo-differential operators. In Sec. 4, complex analysis of the transfer functions is carried out: branching points are computed, from which different choices of cuts are made. Our extensions of diffusive representations are built on these cuts. In Sec. 5, two methods for finite-order approximations are explained and numerical results are presented in the frequency domain. Section 6 presents the input-ouput representation of a whole waveguide thanks to a KellyLochbaum structure which yields "simulable digital waveguides". Finally, Sec. 7 is dedicated to perspectives and open questions.

\section{Input-Output Representation of a Lossy Flared Acoustic Waveguide}

\subsection{Webster-Lokshin model}

A mono-dimensional model of linear acoustic propagation in axisymmetric waveguides, which includes visco-thermal losses at the wall, has been derived assuming the quasi-sphericity of isobars near the wall. ${ }^{3}$ For a fluid at rest until the initial time $t_{0}=0$, the acoustic pressure $p$ and the particle velocity $v$ are governed by $(t \geq 0)$

$$
\begin{aligned}
{\left[\left(\frac{1}{c_{0}^{2}} \partial_{t}^{2}+\frac{2 \varepsilon(\ell)}{c_{0}^{3 / 2}} \partial_{t}^{3 / 2}+\Upsilon(\ell)\right)-\partial_{\ell}^{2}\right][r(\ell) p(\ell, t)] } & =0, \\
\rho_{0} \partial_{t} v(\ell, t)+\partial_{\ell} p(\ell, t) & =0,
\end{aligned}
$$

where $t \geq 0$ is the time variable, $\ell \in[0, L]$ is the space variable measuring the arclength of the wall but not $z$ the usual axis coordinate, $\partial_{t}^{3 / 2}$ is the fractional time derivative $^{4}$ associated in the Laplace domain to the symbol $s^{3 / 2}$ understood with

a cut on $\mathbb{R}^{-}, r(\ell)$ is the radius of the pipe, $\varepsilon(\ell)=\kappa_{0} \frac{\sqrt{1-r^{\prime}(\ell)^{2}}}{r(\ell)}$ quantifies the effect of the visco-thermal losses, and $\Upsilon(\ell)=r^{\prime \prime}(\ell) / r(\ell)$ accounts for the curvature of the pipe. The associated boundary conditions will be precised in the following.

Note that if $\underline{r}(z)$ denotes the radius of the pipe for the $z$-ordinate, the length of the pipe from 0 to $z$ is $\ell(z)=\int_{0}^{z} \sqrt{1+\underline{r}^{\prime}(z)^{2}} \mathrm{~d} z$ and $\underline{r}(z)=r(\ell(z))$. As a consequence, an important property of $r$ is that ${ }^{3}\left|r^{\prime}(\ell)\right| \leq 1\left(r^{\prime}(\ell)=1\right.$ means that $\left.\underline{r}^{\prime}(z) \rightarrow+\infty\right)$.

Physical constants. For standard conditions and for the air at rest, the physical constants are given by: the mass density $\rho_{0}=1.2 \mathrm{~kg} \mathrm{~m}^{-3}$, the speed of sound $c_{0}=344 \mathrm{~m} \mathrm{~s}^{-1}$, the coefficient $\kappa_{0}=\sqrt{l_{v}^{\prime}}+(\gamma-1) \sqrt{l_{h}} \approx 3.510^{-4} \mathrm{~m}^{1 / 2}$ where 
$l_{v}^{\prime}=\mu /\left(\rho_{0} c_{0}\right) \approx 4.39 \times 10^{-8} \mathrm{~m}$ and $l_{h}=\lambda /\left(\rho_{0} c_{0} C_{P}\right) \approx 7.26 \times 10^{-8} \mathrm{~m}$ denote characteristic lengths of viscous and thermal effects ${ }^{5}$ respectively; the heat coefficients at constant pressure and constant volume per unit of mass $C_{P} \approx 1000 \mathrm{~J} \mathrm{~kg}^{-1} \mathrm{~K}^{-1}$ and $C_{V}$ with the specific heat ratio $\gamma=C_{P} / C_{V}=1.4$, the coefficient of shear viscosity $\mu \approx 1.8 \times 10^{-5} \mathrm{~kg} \mathrm{~s}^{-1} \mathrm{~m}^{-1}$, the coefficient of thermal conductivity $\lambda \approx 0.03 \mathrm{~W} \mathrm{~m}^{-1} \mathrm{~K}^{-1}$.

\subsection{Travelling waves and quadripoles of conversion}

An alternative to the acoustic state $(p, v)$ is the state $\left(\psi^{+}, \psi^{-}\right)$, with

$$
\psi^{ \pm}(\ell, t)=\frac{r(\ell)}{2}\left[p(\ell, t) \pm \rho_{0} c_{0} v(\ell, t)\right] \mp \frac{r^{\prime}(\ell)}{2} c_{0} \partial_{t}^{-1} p(\ell, t) .
$$

This change of functions is associated with the reciprocal change:

$$
\begin{aligned}
p(\ell, t) & =\frac{1}{r(\ell)}\left[\psi^{+}(\ell, t)+\psi^{-}(\ell, t)\right], \\
v(\ell, t) & =\frac{1}{r(\ell)} \frac{1}{\rho_{0} c_{0}}\left[\psi^{+}(\ell, t)-\psi^{-}(\ell, t)+\frac{r^{\prime}(\ell)}{r(\ell)} c_{0} \partial_{t}^{-1}\left[\psi^{+}(\ell, t)+\psi^{-}(\ell, t)\right]\right],
\end{aligned}
$$

where $\partial_{t}^{-1} X(\ell, t)=\int_{0}^{t} X(\ell, \tau) \mathrm{d} \tau$ denotes the causal time integration.

This acoustic state defines travelling waves $\psi^{+}$and $\psi^{-}$which extend the usual decoupled incoming and outgoing planar or spherical waves propagating respectively in straight or conical non-visco-thermal pipes $(\varepsilon=0, \Upsilon=0)$. The governing equations (1) and (2) are proven to be equivalent to

$$
\left[\partial_{t} \pm c_{0} \partial_{\ell}\right] \psi^{ \pm}(\ell, t)=-\left[\varepsilon(\ell) \sqrt{c_{0}} \partial_{t}^{1 / 2}+\frac{\Upsilon(\ell) c_{0}^{2}}{2} \partial_{t}^{-1}\right]\left(\psi^{+}(\ell, t)+\psi^{-}(\ell, t)\right) .
$$

The transport operators $\partial_{t} \pm c_{0} \partial_{\ell}$ still make $\psi^{+}$and $\psi^{-}$appear as outwardly and inwardly directed travelling waves, respectively. The right-hand side of Eq. (6) exhibits the coupling between these waves, due to curvature $\Upsilon$ and visco-thermal $\operatorname{losses} \varepsilon$. It is a purely time-operator acting on the total acoustic pressure through $\psi^{+}+\psi^{-}$.

The changes of functions (3)-(5) can also be defined in the Laplace domain, $s$ denoting the Laplace variable (see the definition in Sec. 2.4.1). For the impedance convention, this yields the conversion quadripoles represented in Fig. 1 where $\zeta_{\ell} \triangleq c_{0} r^{\prime}(\ell) / r(\ell)$ is finite for nonzero radius since $\left|r^{\prime}(\ell)\right| \leq 1$.

\subsection{Adimensional variables and equivalent problem for an elementary curved piece of waveguide}

Now, let us consider that the piece of waveguide $\ell \in[0, L]$ is not much curved or is short enough to consider that $\varepsilon(\ell) \approx \varepsilon$ and $\Upsilon(\ell) \approx \Upsilon$ are constant. Defining the adimensional variables $\underline{\boldsymbol{t}}$ and $\underline{\boldsymbol{\ell}}$ as detailed in Table 1 transforms (1), (2) and (6) 


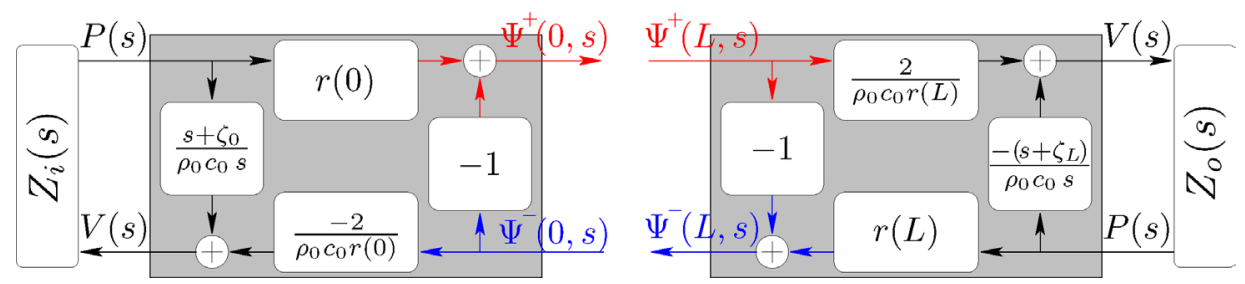

Fig. 1. Conversion quadripoles at the input $(\ell=0$, left $)$ and the output $(\ell=L$, right) of pipe. The impedances $Z_{i}$ and $Z_{0}$ model the boundary conditions.

Table 1. Definition of the adimensional variables and coefficients.

\begin{tabular}{llll}
\hline \multicolumn{2}{c}{ Original problem } & \multicolumn{1}{c}{$\begin{array}{c}\text { Adimensional problem } \\
\text { case } \Upsilon=0\end{array}$} & \multicolumn{1}{c}{$\begin{array}{c}\text { Adimensional problem } \\
\text { case } \Upsilon \neq 0\end{array}$} \\
\hline variable & (unit) & adimensional variable & adimensional variable \\
$\ell$ arclength & $(\mathrm{m})$ & $\underline{\boldsymbol{\ell}}=\ell / L$ & $\underline{\boldsymbol{\ell}}=\ell / L$ \\
$t$ time & $(\mathrm{s})$ & $\underline{\boldsymbol{t}}=t\left(c_{0} / L\right)$ & $\underline{\boldsymbol{t}}=t\left(c_{0} \sqrt{|\Upsilon|}\right)$ \\
$s$ Laplace variable & $\left.\mathrm{s}^{-1}\right)$ & $\underline{\boldsymbol{s}}=-\underline{\boldsymbol{\xi}}+i \underline{\boldsymbol{\omega}}=s\left(L / c_{0}\right)$ & $\underline{\boldsymbol{s}}=s /\left(c_{0} \sqrt{|\Upsilon|}\right)$ \\
& & adimensional coefficient & adimensional coefficient \\
& $\tau=1$ & $\tau=\sqrt{|\Upsilon|} L$ \\
& $\beta=\varepsilon \sqrt{L}$ & $\beta=\varepsilon / \sqrt[4]{|\Upsilon|}$ \\
& $\eta=\Upsilon L^{2}=0$ & $\eta=\operatorname{sgn}(\Upsilon)= \pm 1$ \\
\hline
\end{tabular}

respectively into the convenient forms:

$$
\begin{gathered}
{\left[\partial_{\underline{\underline{\ell}}}^{2}+\tau^{2}\left(\partial_{\underline{\boldsymbol{t}}}^{2}+2 \beta \partial_{\underline{\boldsymbol{t}}}^{3 / 2}+\eta\right)\right][\underline{\boldsymbol{r}}(\underline{\boldsymbol{\ell}}) \underline{\boldsymbol{p}}(\underline{\boldsymbol{\ell}}, \underline{\boldsymbol{t}})]=0,} \\
\left.\rho_{0} c_{0} \tau \partial_{\underline{\boldsymbol{t}}} \underline{\boldsymbol{\ell}}, \underline{\boldsymbol{t}}\right)+\partial_{\underline{\ell}} \underline{\boldsymbol{p}}(\underline{\boldsymbol{\ell}}, \underline{\boldsymbol{t}})=0, \\
\left.\left[\tau \partial_{\underline{\boldsymbol{t}}} \pm \partial_{\underline{\boldsymbol{\ell}}}\right] \underline{\boldsymbol{\psi}^{ \pm}}(\underline{\boldsymbol{\ell}}, \underline{\boldsymbol{t}})=-\tau\left[\beta \partial_{\underline{\boldsymbol{t}}}^{1 / 2}+(\eta / 2) \partial_{\underline{\boldsymbol{t}}}^{-1}\right] \underline{\left(\boldsymbol{\psi}^{+}\right.}(\underline{\boldsymbol{\ell}}, \underline{\boldsymbol{t}})+\underline{\boldsymbol{\psi}^{-}}(\underline{\boldsymbol{\ell}}, \underline{\boldsymbol{t}})\right),
\end{gathered}
$$

for $\underline{\boldsymbol{t}} \geq 0, \underline{\boldsymbol{\ell}} \in[0,1]$ and where $\beta \geq 0$ and $\eta=\operatorname{sgn}(\Upsilon)$.

These adimensional variables are those used in the following but, at each step, all the results can be "denormalized" making use of Table 1. For the sake of legibility, the notation $\underline{X}$ is given up and redefined as $X$.

In the following, only the cases of non-curved $(\eta=0)$ or flared $(\eta=1)$ pipes are considered. The case $\eta=-1$ is discarded from our analysis for reasons detailed in Sec. 7.

\subsection{Scattering matrix and operators of propagation}

\subsubsection{Definitions: Laplace transforms, convolution operators and systems}

We consider the Laplace transform for the causal functions and distributions, defined by $T L[h](s)=\int_{0}^{+\infty} h(t) \mathrm{e}^{-s t} \mathrm{~d} t$. The convergence strip will be $\mathbb{C}_{\alpha}^{+}=\{s \in$ $\mathbb{C} \mid \operatorname{Re}(s)>\alpha\}$ for $\alpha \in \mathbb{R}$.

We denote with cursive capitals such as $\mathcal{H}$, the convolution operators $\mathcal{H}: L^{2}\left(\mathbb{R}^{+}\right) \rightarrow L^{2}\left(\mathbb{R}^{+}\right), u \mapsto y=\mathcal{H} u=h \star u$ with the corresponding convolution 
kernels $h \in L^{1}\left(\mathbb{R}^{+}\right)$which are denoted with lowercase letters. The causal Laplace transforms $H(s)=T L[h](s)$ are denoted with capital letters. They correspond to the "symbol of the convolution operator $\mathcal{H}$ ", but we prefer to call them transfer functions as used in the field of control theory.

Matrices and vectors are denoted with bold letters.

\subsubsection{Webster-Lokshin scattering matrix}

For zero initial conditions, Eq. (9) writes in the Laplace domain, for $s \in \mathbb{C}_{0}^{+}$,

$$
\begin{aligned}
\partial_{\ell} \boldsymbol{\Psi}(\ell, s) & =\boldsymbol{\Theta}(s) \boldsymbol{\Psi}(\ell, s), \\
\boldsymbol{\Theta}(s) & =\tau s\left[\begin{array}{rr}
-1 & 0 \\
0 & 1
\end{array}\right]+\tau(\beta \sqrt{s}+\eta /(2 s))\left[\begin{array}{rr}
-1 & -1 \\
1 & 1
\end{array}\right],
\end{aligned}
$$

where $\boldsymbol{\Psi}(\ell, s)=\left[\Psi^{+}(\ell, s), \Psi^{-}(\ell, s)\right]^{\mathrm{T}}$. The solution of this problem can be represented, thanks to a quadripole, as a 2 input- 2 output system. To define a causal quadripole $\mathbf{Q}$, the inputs and outputs are chosen according to the direction of propagation of $\psi^{ \pm}$. This is a crucial property to allow simulations in the time domain and real-time applications. Inputs are $\left[\psi^{+}(\ell=0, t), \psi^{-}(\ell=1, t)\right]$ and outputs are $\left[\psi^{+}(\ell=1, t), \psi^{-}(\ell=0, t)\right]$, so that the scattering matrix $\mathbf{Q}(s)$ is defined by $\left[\Psi^{+}(1, s), \Psi^{-}(0, s)\right]^{\mathrm{T}}=\mathbf{Q}(s)\left[\Psi^{+}(0, s), \Psi^{-}(1, s)\right]^{\mathrm{T}} \cdot \mathbf{Q}(s)$ is a symmetric matrix given by

$$
\mathbf{Q}(s)=\left[\begin{array}{cc}
T(s) \mathrm{e}^{-\tau s} & R(s) \\
R(s) & T(s) \mathrm{e}^{-\tau s}
\end{array}\right]
$$

(see Appendix A for a complete proof). The corresponding system is represented in Fig. 2. The transmission function $T(s)$ and the reflection function $R(s)$ are

$$
\begin{aligned}
& T(s)=\frac{1-E(s)^{2}}{1-E(s)^{2} D(s)^{2} \mathrm{e}^{-2 \tau s}} D(s), \\
& R(s)=-\frac{1-D(s)^{2} \mathrm{e}^{-2 \tau s}}{1-E(s)^{2} D(s)^{2} \mathrm{e}^{-2 \tau s}} E(s),
\end{aligned}
$$

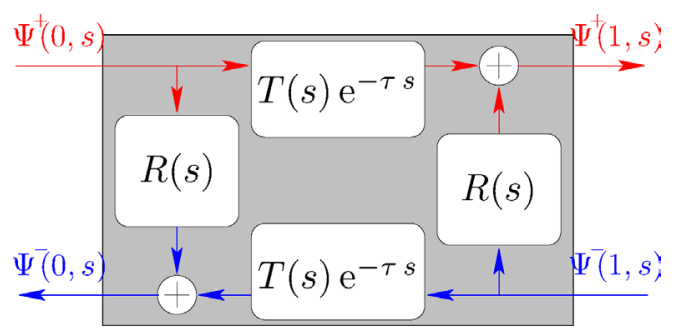

Fig. 2. Representation of the quadripole $\mathbf{Q}$. 
where

$$
\begin{aligned}
E(s) & =\frac{\Gamma(s)-s}{\Gamma(s)+s}, \\
D(s) & =\mathrm{e}^{-\tau(\Gamma(s)-s)}
\end{aligned}
$$

and $\Gamma(s)$ is a square-root of

$$
\Gamma(s)^{2}=s^{2}+2 \beta s^{3 / 2}+\eta .
$$

Taking $-\Gamma(s)$ instead of $\Gamma(s)$ leaves $T(s)$ and $R(s)$ unchanged. In the following, we choose to define $\Gamma(s)$ for $s \in \mathbb{C}_{0}^{+}(\eta \neq-1)$, as the unique analytic continuation of "the positive square-root of Eq. (17) for $s \in \mathbb{R}^{+}$". The analyticity over $\mathbb{C}_{0}^{+}$holds because $\Gamma(s)$ has no branching points in $\mathbb{C}_{0}^{+}$(see the proof in Sec. 4.1.1 for $\eta=1$, the case $\eta=0$ is straightforward). For this definition of $\Gamma(s)$, functions $E(s)$ and $D(s)$ are analytic and such that $|E(s)|<1$ and $|D(s)|<1$ in $\mathbb{C}_{0}^{+}$(see the proof in Appendix B). Moreover, they define causal systems (see the proof in Appendix C). The analyticity in $\mathbb{C}_{0}^{+}$holds also for $T(s)$ and $R(s)$ as well as the causality for their associated systems.

\subsection{Transfer function of a baffled bell}

This section details the case of a baffled bell with a $\mathcal{C}^{1}$-profile and nearly constant parameters $\eta$ and $\beta$, excited by an ideal generator of pressure. The baffle corresponds to $(\beta, \eta)=(0,0)$ so that $\psi^{+}$and $\psi^{-}$are decoupled and propagate as spherical waves. The boundary condition at the output is then $\psi^{-}(1, t)=0$. The ideal generator means that there is no impedance $Z_{0}$ in Fig. 1 . Defining the input $U(s) \triangleq r(0) P(0, s)$, the output $Y(s) \triangleq r(1) P(1, s)$ and deriving the transfer function $F(s)=Y(s) / U(s)$ yields

$$
\begin{aligned}
F(s) & =\frac{T(s) \mathrm{e}^{-\tau s}}{1+R(s)}=\frac{G(s) \mathrm{e}^{-\tau s}}{1-K(s) \mathrm{e}^{-2 \tau s}}, \\
G(s) & =(1+E(s)) D(s)=\frac{2 \Gamma(s)}{\Gamma(s)+s} \mathrm{e}^{-\tau(\Gamma(s)-s)}, \\
K(s) & =-E(s) D(s)^{2}=-\frac{\Gamma(s)-s}{\Gamma(s)+s} \mathrm{e}^{-2 \tau(\Gamma(s)-s)},
\end{aligned}
$$

where $F(s), G(s), K(s)$ are analytic functions in $\mathbb{C}_{0}^{+}$. They define causal systems (see Appendix C). The input-output system associated to $F(s)$ takes the form

$$
Y(s)=G(s) \mathrm{e}^{-\tau s} U(s)+\mathrm{e}^{-2 \tau s} K(s) Y(s) .
$$

It corresponds to the feedback interconnection of subsystems presented in Fig. 3. 
Th. Hélie Es D. Matignon

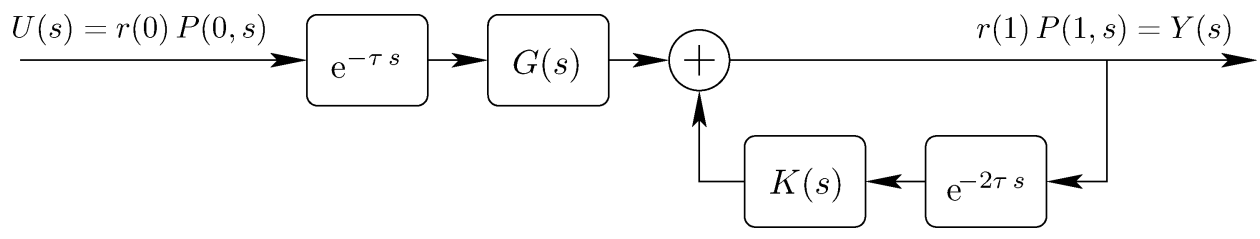

Fig. 3. Block diagram of the system defined by $Y(s) / U(s)=F(s)$.

\subsection{Wavetrain decomposition and discussion on the simulation}

Since $|K(s)|<\mathrm{e}^{-\alpha}<1$ holds for any $s \in \mathbb{C}_{\alpha}^{+}$with $\alpha>0$ (see Sec. B.4), the equality $\left[1-K(s) \mathrm{e}^{-2 \tau s}\right]^{-1}=\sum_{n=0}^{+\infty} K(s)^{n} \mathrm{e}^{-2 n \tau s}$ holds uniformly in $\mathbb{C}_{\alpha}^{+}$. Hence, from Dupraz ${ }^{6}$ and after multiplying by $\mathrm{e}^{-\alpha t}$, for $t \in \mathbb{R}^{+}$,

$$
f(t)=g(t-\tau)+g(t-\tau) \star_{t} \sum_{n=1}^{+\infty} k^{\star n}(t-2 n \tau),
$$

where $f, k$ and $g$ are the inverse Laplace transforms of $F, K, G$, respectively. Note that $f, g$ and $k$ belong to $L^{2}\left(\mathbb{R}^{+}\right) \subset L_{\text {loc }}^{2}\left(\mathbb{R}^{+}\right) \subset L_{\text {loc }}^{1}\left(\mathbb{R}^{+}\right)$(see Table 2 in Appendix C); $\forall n \in \mathbb{N}^{*}, k^{\star(n+1)}=k \star k^{\star n} \in L_{\mathrm{loc}}^{1}\left(\mathbb{R}^{+}\right)$is the $n$th convolution of $k$.

The kernel $g(t-\tau)$ exhibits the delay of propagation $\tau$ and the scattering due to visco-thermal losses and curvature of the pipe. The kernel $\sum_{n=1}^{+\infty} k^{\star n}(t-2 n \tau)=$ $\sum_{n=1}^{\mathrm{E}\left(\frac{t}{2 \tau}\right)} k^{\star n}(t-2 n \tau) \in L_{\mathrm{loc}}^{1}\left(\mathbb{R}^{+}\right)$expresses the successive reflexions at the boundaries of the bell, where $2 \tau$ corresponds to a back and forth propagation. The multiple convolutions $k^{\star n}$ show that, for each new reflexion, the convolution kernel $k$ is cumulated.

The direct use of Eq. (22) for the numerical simulation in the time-domain will be very time- and memory-consuming because the impulse responses $g(t)$ and $k(t)$ decay very slowly, e.g. as $t^{-3 / 2}$ for straight pipes with visco-thermal losses. ${ }^{7}$ The next section is dedicated to build an efficient method for the simulation in the time domain. The first step of this method consists in representing $G(s)$ and $K(s)$ as the aggregation of first-order differential systems. It is performed from the analysis of the singularities of the transfer functions. In a second step, the so-called diffusive representations allow for finite-dimensional approximations which prove efficient for their simulation in the time domain.

The whole waveguides with complex geometries can be represented, by concatenating quadripoles $\mathbf{Q}_{n}$. In this case, a structure similar to Fig. 3 can be built thanks to systematic algebraic manipulations. This is detailed in Sec. 6.1.

\section{Introduction to Diffusive Representations}

The transfer functions $T(s), R(s), G(s), K(s)$ and $H(s)$ are rational functions of the variables $s, \mathrm{e}^{-\tau s}$, and $\Gamma(s), \mathrm{e}^{-\tau(\Gamma(s)-s)}$. Combining the first two variables leads to standard delay-differential equations, well-adapted to low cost numerical 
simulation in the time domain. On the contrary, $\Gamma(s)$ and $\mathrm{e}^{-\tau(\Gamma(s)-s)}$ correspond to pseudo-differential operators, ${ }^{8}$ which are much more difficult to tackle and simulate. An interesting way to overcome this difficulty is to try to decompose the associated convolution kernels on a family of purely damped exponentials with weights $\mu$, or if necessary, on a family of damped oscillating exponentials. Then, a natural approximation strategy will be to select a finite subset of this family to approximate the kernels and the associated operators, yielding straightforward finite-order differential equations for the simulation.

Diffusive representations provide a mathematical framework for such infinitedimensional decompositions, for which well-posed realizations of diagonal type can be easily derived. They help to transform a nonlocal in time pseudo-differential equation into a first-order differential equation on a Hilbert state-space, which allows for stability analysis. We refer to Staffans ${ }^{9}$ (note that in Sec. 5, standard diffusive representations of the first kind are defined; in Sec. 6, generalized diffusive representations of the second kind are introduced in Ref. 9) for the treatment of completely monotone kernels (also known as Laplace transforms of Stieltjes measure), to Montseny ${ }^{10}$ for diffusive representations of pseudo-differential operators, and to Matignon ${ }^{4}$ for links between fractional differential operators and diffusive representations.

\subsection{Standard diffusive representations and realizations}

Diffusive representations give a framework to represent convolution operators $\mathcal{H}$ : $L^{2}(0, T) \rightarrow L^{2}(0, T), u \mapsto y=h \star u$ for which the associated convolution kernel $h$ is defined by

$$
h(t)=\int_{0}^{+\infty} \mathrm{e}^{-\xi t} \mathbf{1}_{t>0}(t) M(\mathrm{~d} \xi),
$$

where $M(\mathrm{~d} \xi)$ is a measure on $\mathbb{R}^{+}$such that

$$
\int_{0}^{+\infty} \frac{|M|(\mathrm{d} \xi)}{1+\xi}<+\infty
$$

$M$ can be a positive real-valued measure, or a signed real-valued measure, or even a complex-valued measure, hence the presence of the modulus sign in Eq. (24). Applying the dominated convergence theorem, the associated transfer function is

$$
H(s)=\int_{0}^{+\infty} \frac{M(\mathrm{~d} \xi)}{s+\xi}
$$

for $s \in \mathbb{C}_{0}^{+}$. When the measure $M(\mathrm{~d} \xi)=\mu(\xi) \mathrm{d} \xi$ is absolutely continuous with respect to the Lebesgue measure, applying the residues theorem for an adapted Bromwich contour (see Fig. 14(c)) proves that $\mu$ is given by ${ }^{4}$

$$
\mu(\xi)=\frac{1}{2 i \pi}\left[H\left(-\xi+i 0^{-}\right)-H\left(-\xi+i 0^{+}\right)\right] .
$$


A huge family of causal convolution kernels belong to this class and a few examples of transfer functions are recalled and detailed in Appendix $\mathrm{D}\left(s \in \mathbb{C}_{0}^{+}\right)$:

- $M_{1}(\mathrm{~d} \xi)=\sum_{k=0}^{K} \mu_{k} \delta_{\xi_{k}}(\mathrm{~d} \xi)$ leads to $H_{1}(s)=\sum_{k=0}^{K} \frac{\mu_{k}}{s+\xi_{k}}\left(\right.$ for $\left.\operatorname{Re}\left(\xi_{k}\right)>0\right)$,

- $M_{2}(\mathrm{~d} \xi)=\sum_{k=0}^{+\infty} 2 \delta_{\left(k+\frac{1}{2}\right)^{2} \pi^{2}}(\mathrm{~d} \xi)$ leads to $H_{2}(s)=\frac{\tanh (\sqrt{s})}{\sqrt{s}}$

- $M_{3}(\mathrm{~d} \xi)=\frac{\cos (\sqrt{\xi})}{\pi \sqrt{\xi}} \mathrm{d} \xi$ leads to $H_{3}(s)=\frac{\mathrm{e}^{-\sqrt{s}}}{\sqrt{s}}$

- $M_{4}(\mathrm{~d} \xi)=\frac{\sin (a \pi)}{\pi} \xi^{-a} \mathrm{~d} \xi$ leads to $H_{4}(s)=s^{-a}$ (for $0<a<1$ ).

One of the interests of this formulation is to represent a class of infinite-dimensional systems, for which the measure $M$ can be a point measure $\left(H_{1}, H_{2}\right)$ or an absolutely continuous measure $\left(H_{3}, H_{4}\right)$, or a combination of such measures. A second interest is that systems such as $H_{2}, H_{3}, H_{4}$, will be approximated by finite-dimensional diffusive systems $\left(H_{1}\right)$ for numerical simulation. A third interest is that the corresponding operators admit a simple diagonal realization in the sense of systems theory, with state $\phi$ :

$$
\begin{aligned}
\partial_{t} \phi(\xi, t) & =-\xi \phi(\xi, t)+u(t), \quad \xi \in \mathbb{R}^{+} \\
y(t) & =\int_{0}^{+\infty} \phi(\xi, t) M(\mathrm{~d} \xi) .
\end{aligned}
$$

A functional framework is available ${ }^{11}$ to prove that this realization is a well-posed systems in the sense of Salamon ${ }^{12}$ and Weiss.

To sum up, diffusive representations are used to represent the so-called diffusive pseudo-differential systems of the first kind, ${ }^{9,10}$ meaning that singularities lie on $s=-\xi \in \mathbb{R}^{-}$. As a consequence, the state $\phi(\xi, t)$ of the realization has a stable dynamics, since it is subject to the damping factor $\xi$.

\subsection{Extension by derivation}

Every transfer function with a cut on $\mathbb{R}^{-}$does not admit a diffusive representation. This is the case of the fractional derivative of order $b(0<b<1)$ associated to $H_{5}(s)=s^{b}(0<b<1)$, for which it does not exist a measure $M$ which fulfills the well-posedness condition, (24). But its realization can be achieved through that of $H(s) / s=s^{-a}$ with $a=1-b$ and $0<a<1$ thanks to the measure $M_{4}$ and, to compensate, by taking the derivative of the output.

For some transfer functions defined in $s=0$ but for which $H(0) \neq 0$, this extension is performed by representing

$$
\breve{H}(s)=\frac{1}{s}[H(s)-H(0)],
$$

thanks to an appropriate measure $\breve{M}(\mathrm{~d} \xi)$. For this extension, the couple $(H(s), \breve{M})$ is unique provided that $\breve{M}$ contains no Dirac measure $\delta_{0}$ at $\xi=0$. In this case, 
Eqs. (25) and (27) become, respectively,

$$
H(s)=s \int_{0}^{+\infty} \frac{\breve{M}(\mathrm{~d} \xi)}{s+\xi}+H(0)
$$

and

$$
\begin{aligned}
\partial_{t} \phi(\xi, t) & =-\xi \phi(\xi, t)+u(t), \quad \xi>0 \\
y(t) & =\int_{0}^{+\infty} \partial_{t} \phi(\xi, t) \breve{M}(\mathrm{~d} \xi)+H(0) u(t) .
\end{aligned}
$$

This is the case of $H_{6}(s)=\mathrm{e}^{-\sqrt{s}}$ which does not admit a standard diffusive representation, but for which $\left(H_{6}(s)-1\right) / s$ is associated to the measure $\breve{M}_{6}(\mathrm{~d} \xi)=\frac{\sin \sqrt{\xi}}{\pi \xi} \mathrm{d} \xi$.

\subsection{Extension for generalized cuts and singularities: Diffusive representation of the second kind}

For many irrational tranfer functions $H(s)$, the associated convolution kernels $h(t)$ cannot be decomposed on purely damped exponentials $\mathrm{e}^{-\xi t} \mathbf{1}_{t>0}(t)$ with $-\xi \in \mathbb{R}^{-}$, but on oscillating damped exponentials $\mathrm{e}^{\gamma t} \mathbf{1}_{t>0}(t)$ with $\gamma \in \mathcal{C} \subset \mathbb{C}_{0}^{-}$. Such generalizations of diffusive representations of the first kind (either standard or extended by derivation) have been proposed by Staffans ${ }^{9}$ and Matignon. ${ }^{4}$

For instance, let us consider the tranfer function $H_{7}(s)=1 / \sqrt{s^{2}+1}$ with associated convolution kernel ${ }^{13} h(t)=J_{0}(t) \mathbf{1}_{t>0}(t), J_{0}$ denoting the 0 -order Bessel function of the first kind. Its extended diffusive representation has been investigated ${ }^{4,14}$ for the definition $\Gamma(s)=\sqrt{s-i} \sqrt{s+i}$. The cuts $\pm i+\mathbb{R}^{-}$are associated to the

complex conjugate weights $\mu^{ \pm}(\xi)$ which are computed using the Bromwich contour Fig. 4(a) by

$$
\mu^{ \pm}(\xi)=\frac{1}{2 i \pi}\left[H_{7}\left(-\xi \pm i+i 0^{-}\right)-H_{7}\left(-\xi \pm i+i 0^{+}\right)\right]=\frac{1}{\pi} \frac{1}{\sqrt{\xi}} \frac{1}{\sqrt{ \pm 2 i-\xi}} .
$$

As $\mu^{-}=\overline{\mu^{+}}, H_{7}(s)$ admits the realization

$$
\begin{aligned}
\partial_{t} \phi(\xi, t) & =(-\xi+i) \phi(\xi, t)+u(t), \quad \xi>0 \\
y(t) & =\int_{0}^{+\infty} \operatorname{Re}\left[\mu^{+}(\xi) \phi(\xi, t)\right] \mathrm{d} \xi,
\end{aligned}
$$

where the weights fulfill the well-posedness condition ${ }^{9}$

$$
\int_{0}^{+\infty}\left|\frac{\mu^{+}(\xi)}{1+\xi-i}\right| \mathrm{d} \xi+\int_{0}^{+\infty}\left|\frac{\mu^{-}(\xi)}{1+\xi+i}\right| \mathrm{d} \xi<+\infty .
$$

Other examples can be found in the transfer functions associated to linear PDEs (see Duffy $\left.{ }^{15}\right)$. The Webster $(\beta=0)$ and Webster-Lokshin $(\beta>0)$ models lead to such generalized diffusive transfer fonctions through $G(s), K(s)$ and $D(s)$. When $\beta=0, \Gamma(s)$ equals $\sqrt{s^{2}+1}$, so that they can be defined with the same cuts as those of $H_{7}(s)$ and its representation can be derived in the same way. 


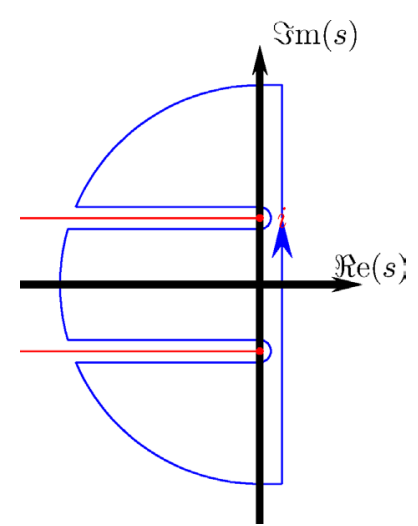

(a)

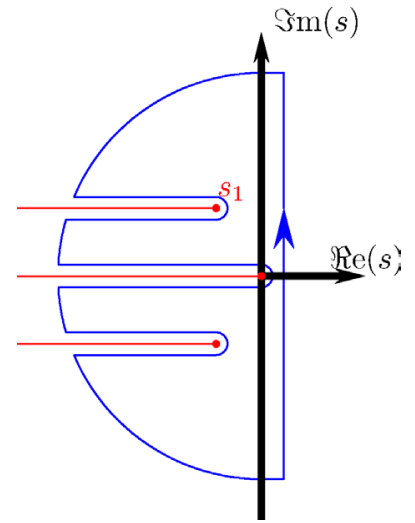

(b)

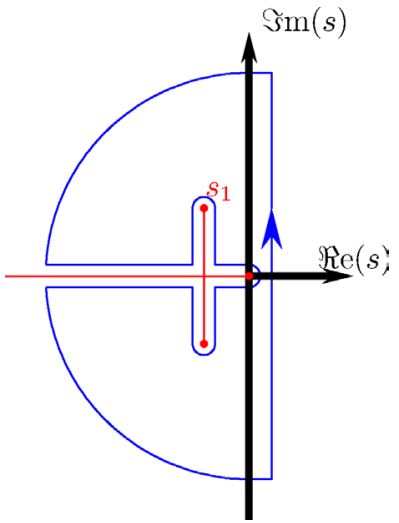

(c)

Fig. 4. Bromwich contours used for deriving the generalized diffusive representations of $H_{7}(s)=$ $1 / \sqrt{s^{2}+1}(\mathrm{a}), G(s)(\mathrm{b})$ and $K(s)(\mathrm{c})$.

In the following, we consider the even more complicated case of the WebsterLokshin transfer functions for which $\Gamma(s)=\sqrt{s^{2}+2 \beta s^{3 / 2}+1}$. This requires both analytical and numerical in-depth studies, and will be presented in the next section.

\section{Diffusive Representations for the Webster-Lokshin Transfer Functions}

In this section, we first perform an analysis of the singularities of the transfer functions in the complex variable $s$, from which we can choose and define cuts between the branching points. In a second step, we adapt the diffusive representation based on the cuts previously chosen.

\subsection{Singularities of the transfer functions $\Gamma(s), G(s)$ and $K(s)$}

\subsubsection{Branching points}

Branching points involved in $\Gamma(s)$ are 0 (because of the term $s^{3 / 2}$ ) and the roots of $\Gamma(s)^{2}$. The roots of $\Gamma(s)^{2}$ are given by $s=\sigma^{2}$ where $\sigma=\rho \mathrm{e}^{i \theta}$ with $-\frac{\pi}{2}<\theta \leq \frac{\pi}{2}$ solves

$$
\sigma^{4}+2 \beta \sigma^{3}+1=0
$$

Two out of the four $\sigma$-roots (see Fig. 5(a)) correspond to complex conjugated branching points, namely, $s_{1}=\sigma_{1}^{2}\left(\operatorname{Im}\left(s_{1}\right) \geq 0\right)$ and $s_{2}=\sigma_{2}^{2}=\overline{s_{1}}$ (see Fig. 5(b)), while the others two do not correspond to $s$-roots.

The transfer functions $G(s)$ and $K(s)$ have three branching points $0, s_{1}(\beta)$ and $\overline{s_{1}} \in \mathbb{C}_{0}^{-}$. The cuts of these functions are naturally the same as those of $\Gamma(s)$. 


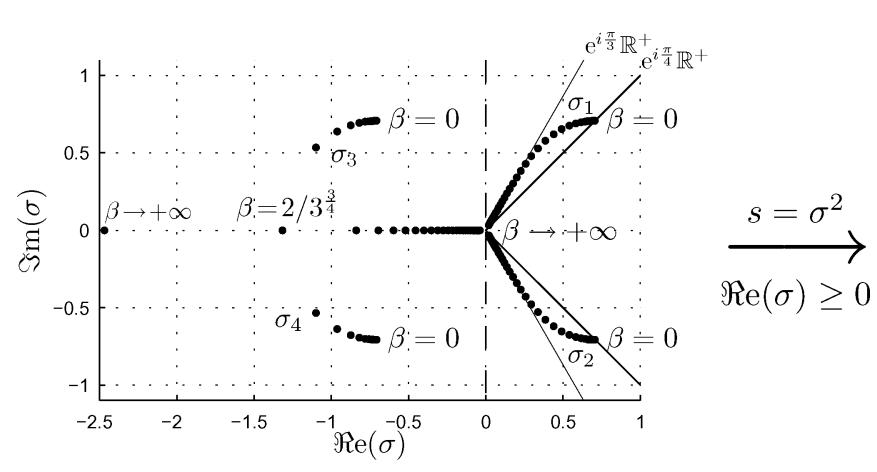

(a)

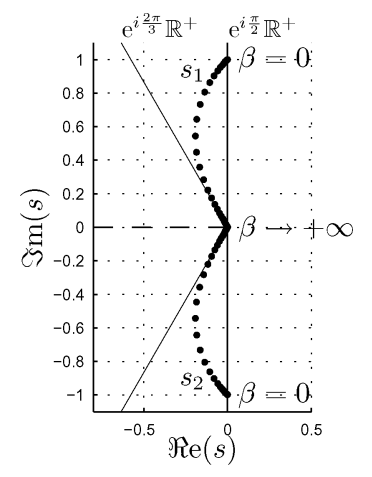

(b)

Fig. 5. The roots of $\sigma^{4}+2 \beta \sigma^{3}+1$ are represented in the complex plane (a). The $\sigma$-roots such that $\operatorname{Re}(\sigma) \geq 0$ have associated $s$-roots represented in (b), namely: $s_{1}=\sigma_{1}{ }^{2}$ and $s_{2}=\sigma_{2}{ }^{2}=\overline{s_{1}}$. These complex conjugate branching-points evolve from $s_{1}=+i$ for $\beta=0$ to $s_{1}=0$ for $\beta \rightarrow+\infty$ with an increasing angle starting from $\pi / 2$ to $2 \pi / 3$, so that they all have a negative real-part (stability domain). The other $\sigma$-roots $\left(\sigma_{3,4}\right)$ have a negative real-part so that they do not correspond to $s$-roots. They evolve as complex conjugate pairs for $0 \leq \beta<2 \times 3^{-3 / 4}$, merge in $\sigma=-\sqrt[4]{3}$ for $\beta=2 \times 3^{-3 / 4}$, then move on the negative real axis towards $-\infty$ for one of them, and towards $0^{-}$ for the other.

\subsubsection{Defining cuts}

As $\Gamma(s)$ is analytic in $\mathbb{C}_{0}^{+}$, the cuts of the possible analytic continuations over $\mathbb{C}$ must be chosen in $\mathbb{C}_{0}^{-}$. Moreover, the transfer functions map real inputs to real outputs, a natural choice is thus to preserve the hermitian symmetry in $\mathbb{C}_{0}^{-}$also. The cut associated to the fractional derivative symbol $s^{3 / 2}$ (starting from 0 ) is $\mathbb{R}^{-}$. But there are infinitely many choices of symmetrical cuts starting from $s_{1}(\beta)$ and $\overline{s_{1}}$. Here, we require cuts to be half-lines. Note that, for instance, this is not the case for the natural definition $\Gamma(s)=\sqrt{s^{2}+2 \beta s^{3 / 2}+1}$ where $X \mapsto \sqrt{X}$ is defined with the cut on $\mathbb{R}^{-}$. Solving $s^{2}+2 \beta s^{3 / 2}+1=-\xi \in \mathbb{R}^{-}$yields two roots $s(\xi)$ and $\overline{s(\xi)}$ which describe two curves in $\mathbb{C}_{0}^{-}$, starting from $s_{1}$ and $\overline{s_{1}}$ respectively (see Fig. 6(a)). But these are not straight lines.

Here, requiring half-lines leads to define the following family

$$
\Gamma(s)=\frac{1}{2}\left[\sqrt[\left(\theta_{1}\right)]{s-s_{1}} \sqrt[\left(\theta_{2}\right)]{s-\overline{s_{1}}} \sqrt{\chi(\sqrt{s})}+\sqrt[\left(\theta_{1}\right)]{\bar{s}-s_{1}} \sqrt[\left(\theta_{2}\right)]{\bar{s}-\overline{s_{1}}} \sqrt{\chi(\sqrt{\bar{s}})}\right],
$$

where $\sqrt[\left(\theta_{0}\right)]{\rho \mathrm{e}^{i \theta}} \triangleq \sqrt{\rho} \mathrm{e}^{i \frac{\theta}{2}}$ with $\rho \in \mathbb{R}^{+}$and $\theta \in\left[\theta_{0}, \theta_{0}+2 \pi[\right.$ and where

$$
\chi(\sigma)=\frac{\left(\sigma-\sigma_{3}\right)\left(\sigma-\sigma_{4}\right)}{\left(\sigma+\sigma_{1}\right)\left(\sigma+\sigma_{2}\right)},
$$

is proven to be such that $\forall \sigma \in \mathbb{C}_{0}^{+}, \chi(\sigma) \in \mathbb{C}_{0}^{+}\left(\sigma_{1,2,3,4}\right.$ are defined in Fig. 5(a)). Thus, in Eq. (35), the term $\sqrt[\left(\theta_{1}\right)]{s-s_{1}} \sqrt[\left(\theta_{2}\right)]{s-\overline{s_{1}}} \sqrt{\chi(\sqrt{s})}$ uniquely defines an analytic continuation of $s \mapsto \Gamma(s)$ on $\mathbb{C}$ for the cuts $\mathbb{R}^{-} \cup\left(s_{1}+\mathrm{e}^{i \theta_{1}} \mathbb{R}^{+}\right) \cup\left(\overline{s_{1}}+\mathrm{e}^{i \theta_{2}} \mathbb{R}^{+}\right)$. The mean of the two terms fulfills the hermitian symmetry. 


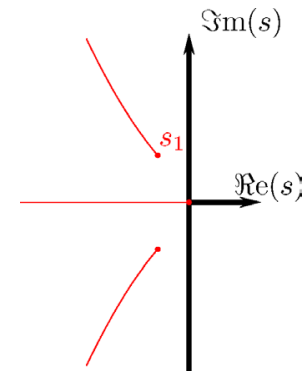

(a)

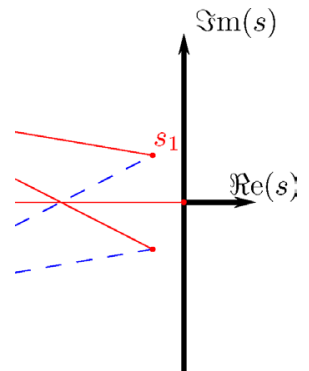

(b)

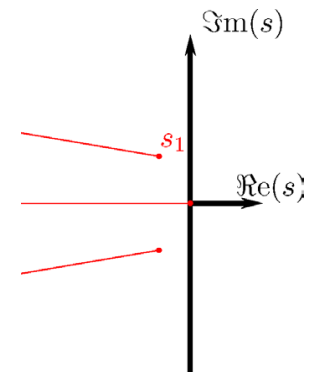

(c)

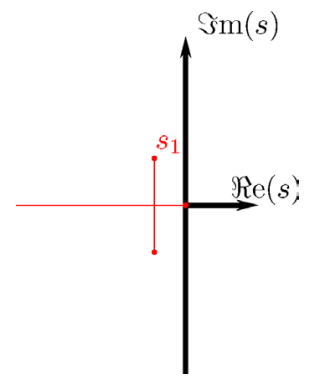

(d)

Fig. 6. These figures illustrate the cuts defined by $\Gamma(s)=\sqrt[(-\pi)]{\Gamma(s)^{2}}$ (a) and by Eq. (35)(b)-(d). If $\theta_{1}, \theta_{2}$ and distinct angles, $\Gamma(s)$ has five cuts: two starting from $s_{1}$, two from $\overline{s_{1}}$, and $\mathbb{R}^{-}$from 0 (b). If $\theta_{1}=-\theta_{2}[2 \pi]$ the two terms of Eq. (35) are identical and $\Gamma(s)$ has only three cuts: one from each branching point (c). Choosing $\theta_{1}=\theta_{2}=-\pi / 2$, the discontinuity of $\sqrt[\left(-\frac{\pi}{2}\right)]{s-\overline{s_{1}}}$ compensates with that of $\sqrt[\left(-\frac{\pi}{2}\right)]{s-s_{1}}$ on $s_{1}-i \mathbb{R}^{+}$and leads to the particular cuts $\mathcal{C}_{c}=\mathbb{R}^{-} \cup\left[s_{1}, \overline{s_{1}}\right]$ (d).

To ensure that the cuts $\mathrm{e}^{ \pm i \theta_{1}} \mathbb{R}^{+}$and $\mathrm{e}^{ \pm i \theta_{2}} \mathbb{R}^{+}$belong to $\mathbb{C}_{0}^{-}$and that $\forall x \in \mathbb{R}^{+}$, $\sqrt[\left(\theta_{0}\right)]{x}=+\sqrt{x}$, the angles must be chosen as follows:

$$
\left(\theta_{1}, \theta_{2}\right) \in \Theta^{2} \quad \text { with } \Theta=\left[-\frac{3 \pi}{2},-\frac{\pi}{2}[+4 \pi \mathbb{Z} .\right.
$$

These functions usually have five cuts (Fig. 6(b)). Choosing $\theta_{1}=-\theta_{2}[2 \pi]$ leads to three cuts (Fig. 6(c)), and the very special case $\theta_{1}=\theta_{2}=-\pi / 2$ leads to the "cross-cuts"(Fig. 6(d)). In the following, only the case of "three horizontal cuts" (case (c) with $\theta_{1}=\theta_{2}=-\pi$ ), denoted $\mathcal{C}_{h}$, and that of the "cross-cuts" (case (d), $\left.\theta_{1}=\theta_{2}=-\pi / 2\right)$, denoted $\mathcal{C}_{c}$, are investigated, with

$$
\begin{aligned}
\mathcal{C}_{h} & =\mathbb{R}^{-} \cup\left(s_{1}+\mathbb{R}^{-}\right) \cup\left(\overline{s_{1}}+\mathbb{R}^{-}\right), \\
\mathcal{C}_{c} & =\mathbb{R}^{-} \cup\left[\overline{s_{1}}, s_{1}\right] .
\end{aligned}
$$

\subsubsection{Poles}

The function $\Gamma(s)$ has no pole. The poles of $G(s)$ and $K(s)$ are the roots of $\Gamma(s)+s$. Their determination is performed in two steps. Firstly, writing $\Gamma(s)^{2}=s^{2}$ leads to solve $2 \beta s^{3 / 2}=1$ and yields $s=(2 \beta)^{-\frac{2}{3}} \mathrm{e}^{ \pm i \frac{2 \pi}{3}}$. Secondly, we check if these complex conjugate solutions are the roots of $\Gamma(s)+s$ (poles of $G(s)$ and $K(s)$ ) or the roots of $\Gamma(s)-s$ (zeros of $K(s)$ ). This result depends on the chosen cuts: they correspond to zeros of $K(s)$ for both the "cross-cuts" $\mathcal{C}_{c}$ and the "horizontal cuts" $\mathcal{C}_{h}$, and to poles for the cuts defined, for example, taking $\theta_{1}=-\frac{3 \pi}{2}, \theta_{2}=-\frac{\pi}{2}$.

The tranfer functions under study in the following have no poles but only cuts.

\subsection{Diffusive representations and realizations of $G(s)$ and $K(s)$}

From this singularities analysis, we build diffusive representations of the transfer functions $G(s)$ and $K(s)$. A first family of representations is built for the cut 
$\mathcal{C}_{h}$, and a second one for $\mathcal{C}_{c}$. For $K(s)$, the computation of weights $\mu$ yields measures $M(\mathrm{~d} \gamma)$ satisfying the well-posedness criterion

$$
\int_{\mathcal{C}}\left|\frac{M(\mathrm{~d} \gamma)}{1-\gamma}\right|<+\infty
$$

for $\mathcal{C}=\mathcal{C}_{h}$ and $\mathcal{C}=\mathcal{C}_{c}$. The case of $G(s)$ requires an extension by derivation.

\subsubsection{Generalized diffusive representations for the cuts $\mathcal{C}_{h}$ and $\mathcal{C}_{c}$}

Applying the residue theorem with the oriented Bromwich contour Fig. 4(b) for $K(s)$ yields, thanks to the dominated convergence theorem,

$$
\begin{aligned}
\forall s \in \mathbb{C}_{0}^{+}, \quad K(s)= & \int_{0}^{+\infty} \frac{\mu_{0, \mathcal{C}_{h}}(\xi)}{s+\xi} \mathrm{d} \xi+\int_{0}^{+\infty} \frac{\mu_{s_{1}, \mathcal{C}_{h}}(\xi)}{s-s_{1}+\xi} \mathrm{d} \xi \\
& +\int_{0}^{+\infty} \frac{\mu_{\overline{s_{1}}, \mathcal{C}_{h}}(\xi)}{s-\overline{s_{1}}+\xi} \mathrm{d} \xi \\
\forall t \in \mathbb{R}, \quad k(t)= & \int_{0}^{+\infty} \mathrm{e}^{-\xi t} \mathbf{1}_{t>0}(t) \mu_{0, \mathcal{C}_{h}}(\xi) \mathrm{d} \xi \\
& +\int_{0}^{+\infty} \mathrm{e}^{\left(s_{1}-\xi\right) t} \mathbf{1}_{t>0}(t) \mu_{s_{1}, \mathcal{C}_{h}}(\xi) \mathrm{d} \xi \\
& +\int_{0}^{+\infty} \mathrm{e}^{\left(\overline{s_{1}}-\xi\right) t} \mathbf{1}_{t>0}(t) \mu_{\overline{s_{1}}, \mathcal{C}_{h}}(\xi) \mathrm{d} \xi,
\end{aligned}
$$

where the weights $\mu_{\alpha, \mathcal{C}_{h}}$ for $\alpha \in\left\{0, s_{1}, \overline{s_{1}}\right\}$ are given by, for $\xi>0$,

$$
\mu_{\alpha, \mathcal{C}_{h}}(\xi)=\frac{K_{\mathcal{C}_{h}}\left(\alpha-\xi+i 0^{-}\right)-K_{\mathcal{C}_{h}}\left(\alpha-\xi+i 0^{+}\right)}{2 i \pi} .
$$

$K_{\mathcal{C}_{h}}(s)$ denotes the analytic extension of $K(s)$ on $\mathbb{C} \backslash \mathcal{C}_{h}$. These weights are detailed in Table 3 and represented in Fig. 7(2),(3).

The representation associated to $\mathcal{C}_{c}$ is obtained with the Bromwich contour Fig. 4(c) and yields

$$
\begin{aligned}
\forall s \in \mathbb{C}_{0}^{+}, \quad K(s)= & \int_{0}^{+\infty} \frac{\mu_{0, \mathcal{C}_{c}}(\xi)}{s+\xi} \mathrm{d} \xi+\int_{-\operatorname{Im}\left(s_{1}\right)}^{\operatorname{Im}\left(s_{1}\right)} \frac{\mu_{s_{1}, \mathcal{C}_{c}}(\omega)}{s-\operatorname{Re}\left(s_{1}\right)-i \omega} \mathrm{d} \omega, \\
\forall t \in \mathbb{R}, \quad k(t)= & \int_{0}^{+\infty} \mathrm{e}^{-\xi t} \mathbf{1}_{t>0}(t) \mu_{0, \mathcal{C}_{c}}(\xi) \mathrm{d} \xi \\
& +\int_{-\operatorname{Im}\left(s_{1}\right)}^{\operatorname{Im}\left(s_{1}\right)} \mathrm{e}^{\left(\operatorname{Re}\left(s_{1}\right)+i \omega\right) t} \mathbf{1}_{t>0}(t) \mu_{s_{1}, \mathcal{C}_{c}}(\omega) \mathrm{d} \omega,
\end{aligned}
$$

where the weights are given by, for $\xi>0$ and $\omega \in\left[-\operatorname{Im}\left(s_{1}\right), \operatorname{Im}\left(s_{1}\right)\right]$

$$
\begin{aligned}
\mu_{0, \mathcal{C}_{c}}(\xi) & =\frac{K_{\mathcal{C}_{c}}\left(-\xi+i 0^{-}\right)-K_{\mathcal{C}_{c}}\left(-\xi+i 0^{+}\right)}{2 i \pi}, \\
\mu_{s_{1}, \mathcal{C}_{c}}(\omega) & =\frac{K_{\mathcal{C}_{c}}\left(\operatorname{Re}\left(s_{1}\right)+i \omega+0^{-}\right)-K_{\mathcal{C}_{c}}\left(\operatorname{Re}\left(s_{1}\right)+i \omega+0^{+}\right)}{2 i \pi} .
\end{aligned}
$$

$K_{\mathcal{C}_{c}}(s)$ is the analytic extension of $K(s)$ for the cuts $\mathcal{C}_{c}$. 


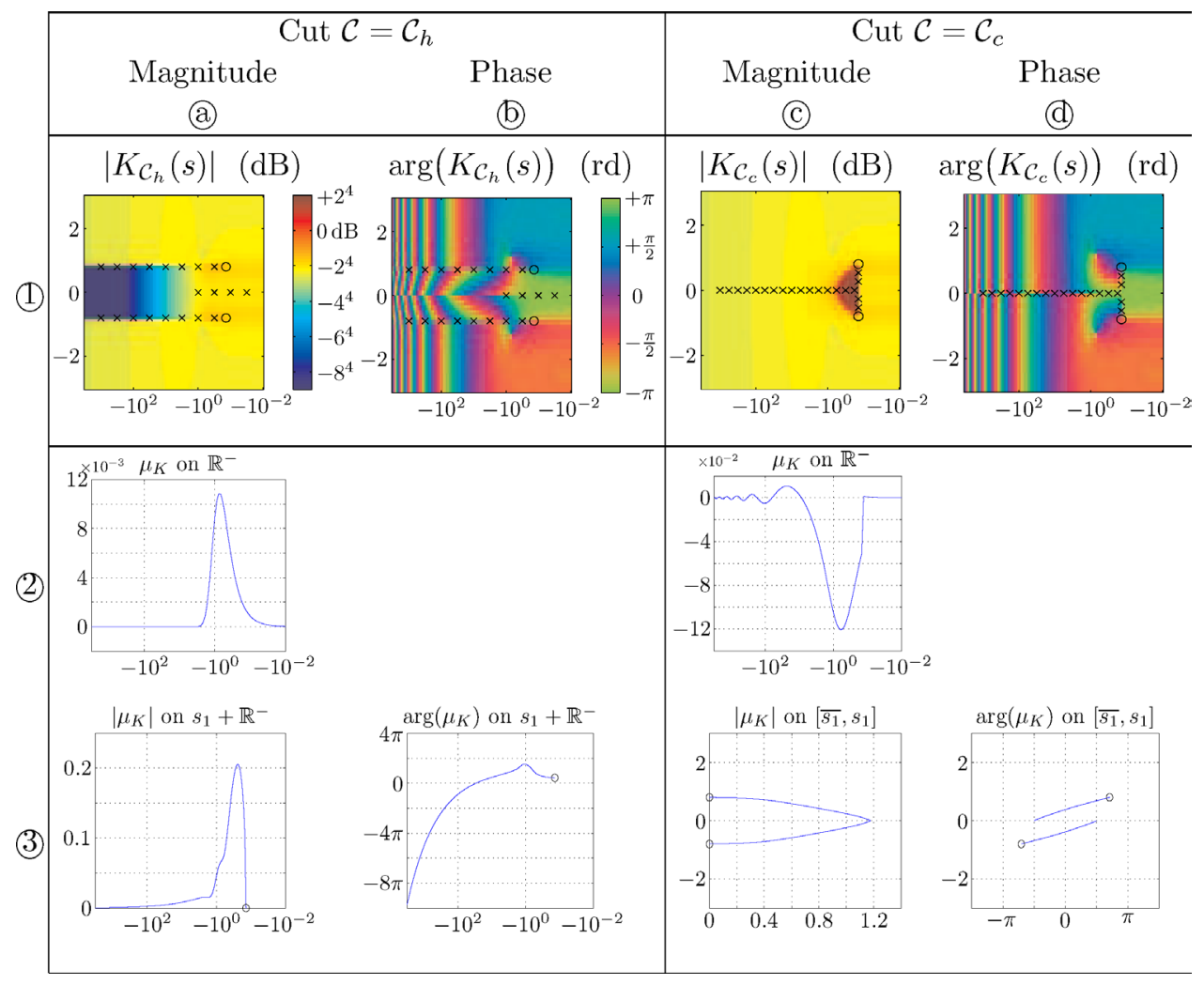

Fig. 7. Modulus and phase of $K(s), s \in \mathbb{C}_{0}^{-}$are represented in (1) for both cuts $\mathcal{C}_{h}$ (a), (b) and $\mathcal{C}_{c}(\mathrm{c}),(\mathrm{d})$. The corresponding (real-valued) weights $\mu$ computed on $\mathbb{R}^{-}$are in (2)a,c. The corresponding (complex-valued) are computed on $s_{1}+\mathbb{R}^{-}$(3)a:modulus, (3)b:phase) and on $\left[\overline{s_{1}}, s_{1}\right]$ (3)c:modulus, (3)d:phase), respectively. The $x$-axis of (1), (2) and (3)a,b correspond to $-\xi=\operatorname{Re}(s)$ $<0$ on a log-scale, and the $y$-Axis of (1), (3)c,d to $\omega=\operatorname{Im}(s)$ on a linear scale. Circles $o$ represent branching points and crosses $\times$ the pole placement for the approximation (see Sec. 5).

These representations satisfy the well-posedness condition, (40). Moreover, because of hermitian symmetry, $\mu_{0, \mathcal{C}_{h}}$ and $\mu_{0, \mathcal{C}_{c}}$ are real-valued, $\mu_{\overline{s_{1}}, \mathcal{C}_{h}}(\xi)=$ $\overline{\mu_{s_{1}, \mathcal{C}_{h}}(\xi)}$ for $\xi>0$ and $\mu_{s_{1}, \mathcal{C}_{c}}(-\omega)=\overline{\mu_{s_{1}, \mathcal{C}_{c}}(\omega)}$ for $\omega \in\left[-\operatorname{Im}\left(s_{1}\right), \operatorname{Im}\left(s_{1}\right)\right]$. Hence, (42) and (45) also take the real-valued form, for $t \in \mathbb{R}$,

$$
\begin{aligned}
k(t)= & \int_{0}^{+\infty} \mathrm{e}^{-\xi t} \mathbf{1}_{t>0}(t) \mu_{0, \mathcal{C}_{h}}(\xi) \mathrm{d} \xi+2 \operatorname{Re} \int_{0}^{+\infty} \mathrm{e}^{\left(s_{1}-\xi\right) t} \mathbf{1}_{t>0}(t) \mu_{s_{1}, \mathcal{C}_{h}}(\xi) \mathrm{d} \xi \\
= & \int_{0}^{+\infty} \mathrm{e}^{-\xi t} \mathbf{1}_{t>0}(t) \mu_{0, \mathcal{C}_{c}}(\xi) \mathrm{d} \xi \\
& +2 \operatorname{Re} \int_{0}^{\operatorname{Im}\left(s_{1}\right)} \mathrm{e}^{\left(\operatorname{Re}\left(s_{1}\right)+i \omega\right) t} \mathbf{1}_{t>0}(t) \mu_{s_{1}, \mathcal{C}_{c}}(\omega) \mathrm{d} \omega
\end{aligned}
$$

Similar calculations for $G(s)$ do not lead to measures fulfilling Eq. (40): The computed weights are not decreasing with $\xi$ (see Fig. 8(2),(3)). This requires to 


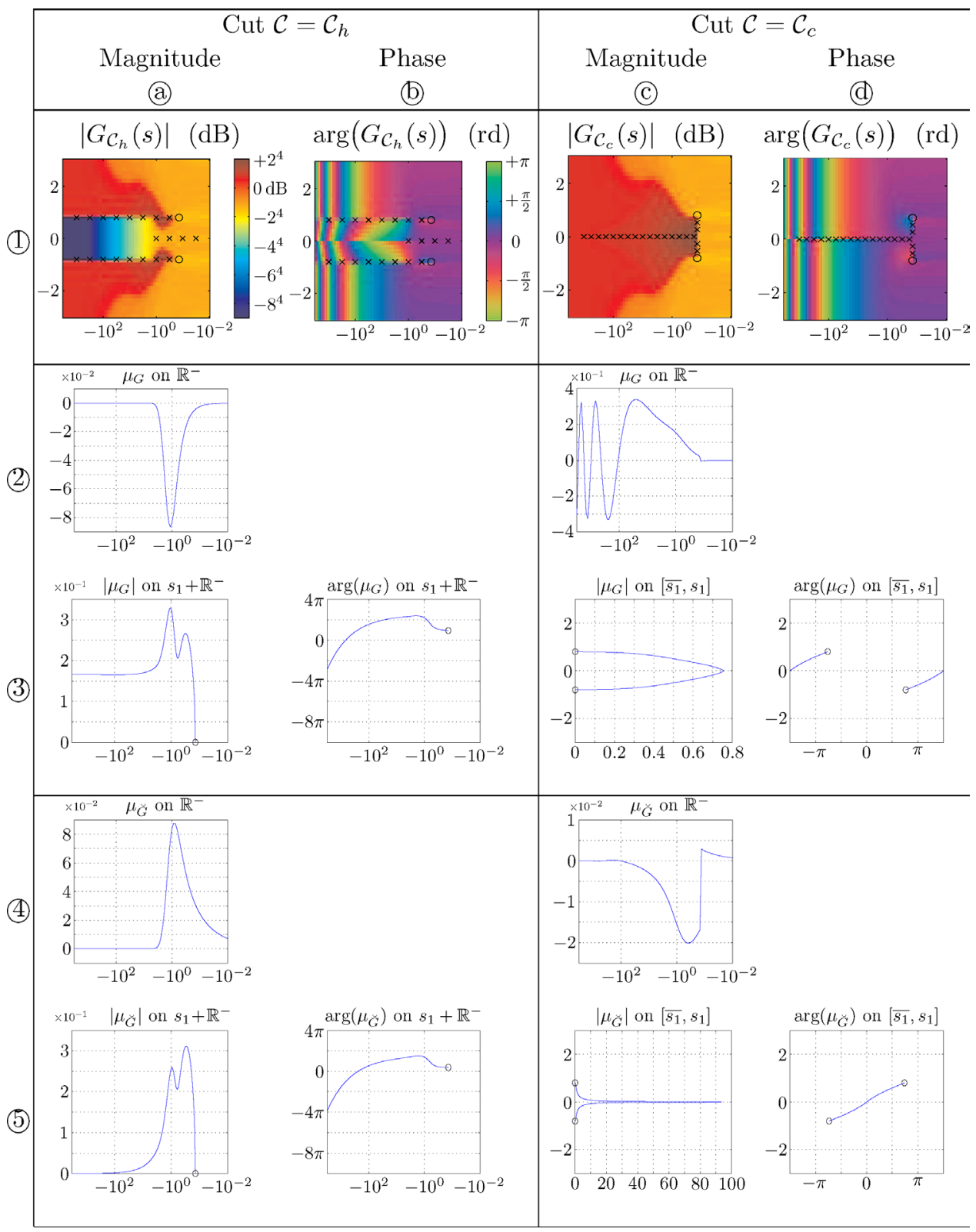

Fig. 8. Figure similar to Fig. 7 for $G(s)$ in (1),(2), (3), and for $\breve{G}(s)$ in (4),(5). The weigths $\mu$ in (2)c and (3)a do not decrease when $-\xi \rightarrow-\infty$ so that the well-posedness condition (40) is not satisfied. This problem disappear in (4), (5) for the extension by derivation computed from $\breve{G}(s)$.

perform an extension by derivation (see Sec. 3.2) computing $\breve{G}(s)=\frac{G(s)-G(0)}{s}$ for both $\mathcal{C}_{h}$ and $\mathcal{C}_{c}$. We check that the well-posedness condition (40) is satisfied for $\breve{G}(s)$. The corresponding weights are given in Table 3 and represented in Fig. 8(4),(5). 
Then, $G(s)$ can be represented by, for $s \in \mathbb{C}_{0}^{+}$,

$$
\begin{aligned}
G(s) & =G(0)+s \breve{G}(s) \\
\breve{G}(s) & =\int_{0}^{+\infty} \frac{\breve{\mu}_{0, \mathcal{C}_{h}}(\xi)}{s+\xi} \mathrm{d} \xi+\int_{0}^{+\infty} \frac{\breve{\mu}_{s_{1}, \mathcal{C}_{h}}(\xi)}{s-s_{1}+\xi} \mathrm{d} \xi+\int_{0}^{+\infty} \frac{\breve{\mu}_{\overline{s_{1}}, \mathcal{C}_{h}}(\xi)}{s-\overline{s_{1}}+\xi} \mathrm{d} \xi \\
& =\int_{0}^{+\infty} \frac{\breve{\mu}_{0, \mathcal{C}_{c}}(\xi)}{s+\xi} \mathrm{d} \xi+\int_{-\operatorname{Im}\left(s_{1}\right)}^{\operatorname{Im}\left(s_{1}\right)} \frac{\breve{\mu}_{s_{1}, \mathcal{C}_{c}}(\omega)}{s-\operatorname{Re}\left(s_{1}\right)-i \omega} \mathrm{d} \omega .
\end{aligned}
$$

The weights are computed, thanks to Eqs. (43), (46) and (47) taking $\breve{G}_{\mathcal{C}_{h}}(s)$ instead of $K_{\mathcal{C}_{h}}(s)$ and $\breve{G}_{\mathcal{C}_{c}}(s)$ instead of $K_{\mathcal{C}_{c}}(s)$.

Choosing cuts yield equivalent diffusive representations but provides distinct interpretations. For $\mathcal{C}_{h}$, the convolution kernels are proven to admit a well-posed decomposition on a family of purely damped exponentials and of damped oscillating exponentials with the pulsation $\operatorname{Im}\left(s_{1}\right)$ (see $\left.(42)\right) . \mathcal{C}_{c}$ is associated to a family of purely damped exponentials and of damped oscillating exponentials with the constant damping factor $-\operatorname{Re}\left(s_{1}\right)$ and with pulsations smaller than $\operatorname{Im}\left(s_{1}\right)$ (see Eq. (45)). More generally, choosing a possible set of cuts is similar to choosing a family of exponentials allowing such decompositions.

\subsubsection{Realizations of the transfer functions in the time domain}

For each cut, an associated time-domain realization of both operators $y=\mathcal{K} u$ and $z=\mathcal{G} u$ can be derived. Those built on the $\mathcal{C}_{h}$-representations are straightforwardly deduced from Eqs. (41), (49) and (50) and are given by

$$
\begin{aligned}
\partial_{t} \phi_{\alpha}(\xi, t)= & (\alpha-\xi) \phi_{\alpha}(\xi, t)+u(t), \quad \xi \in \mathbb{R}^{+}, \alpha \in\left\{0, s_{1}\right\} \\
y(t)= & \int_{0}^{+\infty} \phi_{0}(\xi, t) \mu_{0, \mathcal{C}_{h}}(\xi) \mathrm{d} \xi+2 \operatorname{Re} \int_{0}^{+\infty} \phi_{s_{1}}(\xi, t) \mu_{s_{1}, \mathcal{C}_{h}}(\xi) \mathrm{d} \xi \\
z(t)= & \partial_{t}\left(\int_{0}^{+\infty} \phi_{0}(\xi, t) \breve{\mu}_{0, \mathcal{C}_{h}}(\xi) \mathrm{d} \xi+2 \operatorname{Re} \int_{0}^{+\infty} \phi_{s_{1}}(\xi, t) \breve{\mu}_{s_{1}, \mathcal{C}_{h}}(\xi) \mathrm{d} \xi\right) \\
& +G(0) u(t) .
\end{aligned}
$$

Swapping the time-derivative and the integral operator thanks to Lebesgue's dominated convergence theorem, which applies in the appropriate functional framework, ${ }^{10}$ Eq. (53) is also given by

$$
\begin{aligned}
z(t)= & G(0) u(t)+\int_{0}^{+\infty}\left[-\xi \phi_{0}(\xi, t)+u(t)\right] \breve{\mu}_{0, \mathcal{C}_{h}}(\xi) \mathrm{d} \xi \\
& +2 \operatorname{Re} \int_{0}^{+\infty}\left[\left(s_{1}-\xi\right) \phi_{s_{1}}(\xi, t)+u(t)\right] \breve{\mu}_{s_{1}, \mathcal{C}_{h}}(\xi) \mathrm{d} \xi .
\end{aligned}
$$

The realizations built on the $\mathcal{C}_{c^{-}}$-representations are deduced from Eqs. (44), (49) and (50) and are given by

$$
\begin{aligned}
\partial_{t} \phi_{0}(\xi, t) & =-\xi \phi_{0}(\xi, t)+u(t), \quad \xi \in \mathbb{R}^{+}, \\
\partial_{t} \phi_{s_{1}}(\omega, t) & =\left(\operatorname{Re}\left(s_{1}\right)+i \omega\right) \phi_{s_{1}}(\omega, t)+u(t), \quad \omega \in\left[0, \operatorname{Im}\left(s_{1}\right)\right],
\end{aligned}
$$




$$
\begin{aligned}
y(t)= & \int_{0}^{+\infty} \phi_{0}(\xi, t) \mu_{0, \mathcal{C}_{c}}(\xi) \mathrm{d} \xi+2 \operatorname{Re} \int_{0}^{\operatorname{Im}\left(s_{1}\right)} \phi_{s_{1}}(\omega, t) \mu_{s_{1}, \mathcal{C}_{c}}(\omega) \mathrm{d} \omega, \\
z(t)= & G(0) u(t)+\int_{0}^{+\infty}\left(-\xi \phi_{0}(\xi, t)+u(t)\right) \breve{\mu}_{0, \mathcal{C}_{c}}(\xi) \mathrm{d} \xi \\
& +2 \operatorname{Re} \int_{0}^{\operatorname{Im}\left(s_{1}\right)}\left(\left(s_{1}-\omega\right) \phi_{s_{1}}(\omega, t)+u(t)\right) \breve{\mu}_{s_{1}, \mathcal{C}_{c}}(\omega) \mathrm{d} \omega .
\end{aligned}
$$

\section{Finite Dimensional Approximation and Time-Domain Simulation}

Finite order versions of the stable realizations Eq. (27) are obtained by picking up a finite subset of points which belong to the cuts. Two standard methods yielding such approximations are first recalled. Secondly, numerical results are presented in the frequency domain, for approximations built on both $\mathcal{C}_{h^{-}}$and $\mathcal{C}_{c^{-}}$-representations. Finally, associated low-cost time-domain simulations are proposed and the error of approximation is quantified.

\subsection{Methods of approximation}

\subsubsection{Approximation by interpolation of the state $\phi$}

For a standard diffusive representation (see Sec. 3.1), a first method consists in approximating $\phi(\xi, t)$ by $^{16}$

$$
\tilde{\phi}(\xi, t)=\sum_{k=1}^{K} \phi(\xi, t) \Lambda_{k}(\xi),
$$

where $\left\{\Lambda_{k}\right\}_{1 \leq k \leq K}$ defines continuous piecewise linear interpolating functions nonzero on $] \xi_{k-1}, \xi_{k+1}\left[\right.$ such that $\Lambda_{k}\left(\xi_{k}\right)=1$, with $\xi_{0}<\xi_{1}<\cdots<\xi_{K}<\xi_{K+1}$. The realization (27) is approximated by the first-order linear system of dimension $K$

$$
\begin{aligned}
\partial_{t} \tilde{\phi}_{k}(t) & =-\xi_{k} \tilde{\phi}_{k}(t)+u(t), \\
y(t) & =\sum_{k=1}^{K} \tilde{\mu}_{k} \tilde{\phi}_{k}(t)
\end{aligned}
$$

with

$$
\tilde{\mu}_{k}=\int_{\xi_{k-1}}^{\xi_{k+1}} \mu(\xi) \Lambda_{k}(\xi) \mathrm{d} \xi
$$

A straightforward and similar approximation can be derived for the generalized realizations (51)-(58) interpolating some $\phi(\gamma, t)$ where $\gamma$ lies on the cuts $\mathcal{C}_{h}$ or $\mathcal{C}_{c}$. This method has nice theoretical convergence properties but requires large values of $K$ in practice. 


\subsubsection{Approximation by optimization of a criterion}

A second method consists in a least-square regularized optimization of the weigths $\boldsymbol{\mu}$, by minimizing an appropriate distance between an exact transfer function $H(i \omega)$ and its approximation $\widetilde{H}_{\boldsymbol{\mu}}(i \omega)$ in the frequency domain. For a given order of approximation $K$, this method yields much better results than the interpolation one. ${ }^{17}$ Moreover, the distance can be adapted to optimize some performances, in our context, audio performances.

Model of approximation. Let $\mathcal{S}$ be a finite subset of points included in the cuts $\mathcal{C}_{h}$ or $\mathcal{C}_{c}$, these points being described by $-\xi=\left(-\xi_{j}\right)_{1 \leq j \leq J}, \xi_{j}>0$ for those lying on $\mathbb{R}^{-}$and by $\gamma=\left(\gamma_{k}\right)_{1 \leq k \leq K}$ with $\gamma_{k}=-\xi_{k}^{\prime}+i \omega_{k}^{\prime}, \xi_{k}^{\prime}>0$, and $\omega_{k}^{\prime}>0$ otherwise. The approximation $\widetilde{H_{\mu}}$ is defined by

$$
\widetilde{H}_{\mu}(s)=\sum_{j=0}^{J} \frac{\mu_{j}}{s+\xi_{j}}+\sum_{k=0}^{K}\left[\mu_{k}^{R}\left(\frac{1}{s-\gamma_{k}}+\frac{1}{s-\overline{\gamma_{k}}}\right)+\mu_{k}^{I}\left(\frac{i}{s-\gamma_{k}}+\frac{-i}{s-\overline{\gamma_{k}}}\right)\right],
$$

where $\boldsymbol{\mu}$ denotes the vector $\boldsymbol{\mu} \triangleq\left(\mu_{1}, \ldots, \mu_{J}, \mu_{1}^{R}, \ldots, \mu_{K}^{R}, \mu_{1}^{I}, \ldots, \mu_{K}^{I}\right)^{t} \in \mathbb{R}^{J+2 K}$, $\mu_{j}$ and $\mu_{k}^{\prime}=\mu_{k}^{R}+i \mu_{k}^{I}$ are the associated weights. Requiring real values for $\boldsymbol{\mu}$ ensures hermitian symmetry.

Criterion for audio performances. The criterion to be optimized is

$$
\begin{aligned}
\mathcal{C}(\boldsymbol{\mu})= & \int_{\mathbb{R}^{+}}\left|\left(\widetilde{H_{\mu}}(i \omega)-H(i \omega)\right) w_{H}(\omega)\right|^{2} M(\mathrm{~d} \omega) \\
& +\sum_{j=1}^{J} \epsilon_{j}\left(\mu_{j}\right)^{2}+\sum_{k=1}^{K} \epsilon_{k}^{\prime}\left(\left(\mu_{k}^{R}\right)^{2}+\left(\mu_{k}^{I}\right)^{2}\right) .
\end{aligned}
$$

The parameters $\epsilon_{j} \geq 0$ are regularizing parameters for the purely diffusive part, and $\epsilon_{k}^{\prime} \geq 0$ for the damped oscillating part. They allow to keep the problem wellconditioned when the size $J+2 K$ of $\boldsymbol{\mu}$ increases. The measure $M$ and the weightening $w_{H}$ are chosen according to audio performances and human perception scales ${ }^{18}$ :

(i) Frequencies are perceived from $20 \mathrm{~Hz}$ to $20 \mathrm{kHz}$ on a logarithmic scale. We choose

$$
M(\mathrm{~d} \omega) \triangleq \mathbf{1}_{\omega_{-}<\omega<\omega_{+}}(\omega) \mathrm{d} \ln \omega=\mathbf{1}_{\omega_{-}<\omega<\omega_{+}}(\omega) \frac{\mathrm{d} \omega}{\omega} .
$$

(ii) The perception of intensity is also logarithmic so that we consider the relative error $\left|\widetilde{H_{\mu}}(i \omega)-H(i \omega)\right| /|H(i \omega)|$ rather than the absolute error $\mid \widetilde{H_{\mu}}(i \omega)-$ $H(i \omega) \mid$. This yields the weightening $w_{H}(\omega)=1 /|H(i \omega)|$.

(iii) A precise modelling is sufficient for the typical "audio dynamics" of $80 \mathrm{~dB}$. The previous weightening $w_{H}(\omega)$ can then be revised taking

$$
w_{H}(i \omega)=1 / \operatorname{Sat}_{H, T_{r}}(i \omega) .
$$

The saturation function with treshold $T_{r}$ is defined by $\operatorname{Sat}_{H, T_{r}}(i \omega)$ equals $T_{H} \triangleq$ $T_{r} \sup _{\omega_{-}<\omega<\omega_{+}}|H(i \omega)|$ if $|H(i \omega)|<T_{H}$, and equals $|H(i \omega)|$ otherwise. This 
weightening is finite even for transfer functions with zeros. Note that $80 \mathrm{~dB}$ corresponds to $T_{r}=10^{-4}$.

(iv) In the case of an extension by derivation $\breve{H}$, we need an optimization for $H$ through that of $G$. Hence, the weightening to apply to $\breve{H}$ is then that of $G$ with a compensation of the integrator $1 / s$ in (28), i.e.

$$
\breve{w}_{H}(i \omega)=\omega / \operatorname{Sat}_{H, T_{r}}(i \omega) .
$$

Note that the saturation function is parametrized by $H$ and not $\breve{H}$, but that the weightening $\breve{w}_{H}$ will be applied on $\breve{H}$ in (63).

For numerical computations, the criterion is computed for a finite set of angular frequencies $\left(\omega_{n}\right), 1 \leq n \leq N+1$ increasing from $\omega_{1}=\omega_{-}$to $\omega_{N+1}=\omega_{+}$. It is approximated by

$$
\begin{aligned}
\mathcal{C}(\boldsymbol{\mu})= & \sum_{n=1}^{N}\left|\left(\widetilde{H_{\mu}}\left(i \omega_{n}\right)-H\left(i \omega_{n}\right)\right) w_{H}\left(\omega_{n}\right)\right|^{2}\left[\ln \omega_{n+1}-\ln \omega_{n}\right] \\
& +\sum_{j=1}^{J} \epsilon_{j}\left(\mu_{j}\right)^{2}+\sum_{k=1}^{K} \epsilon_{k}^{\prime}\left(\left(\mu_{k}^{R}\right)^{2}+\left(\mu_{k}^{I}\right)^{2}\right) .
\end{aligned}
$$

It takes the equivalent matrix formulation

$$
\mathcal{C}(\boldsymbol{\mu})=(\mathbf{M} \boldsymbol{\mu}-\mathbf{H})^{*} \mathbf{W}^{*} \mathbf{W}(\mathbf{M} \boldsymbol{\mu}-\mathbf{H})+\boldsymbol{\mu}^{t} \mathbf{E} \boldsymbol{\mu},
$$

where $\mathbf{M}^{*} \triangleq \overline{\mathbf{M}}^{t}$ denotes the transpose conjugate matrix of $\mathbf{M}$. The matrix $\mathbf{M}$ is defined by $M_{n, m}=\left[i \omega_{n}+\xi_{m}\right]^{-1}$ for $1 \leq m \leq J$, by $M_{n, m}=\frac{1}{i \omega_{n}-\gamma_{m-J}}+\frac{1}{i \omega_{n}-\overline{\gamma_{m-J}}}$ for $J+1 \leq m \leq J+K$, and by $M_{n, m}=\frac{i}{i \omega_{n}-\gamma_{m-(J+K)}}+\frac{i}{i \omega_{n}-\gamma_{m-(J+K)}}$ for $J+K+1 \leq$ $m \leq J+2 K$. The vector $\mathbf{H}$ is the column vector $\left(H\left(i \omega_{n}\right)\right)_{1 \leq n \leq N}$. The weightening matrix $\mathbf{W}$ is real positive diagonal and defined by $W_{n, n}=w_{H}\left(\omega_{n}\right) \sqrt{\ln \omega_{n+1}-\ln \omega_{n}}$ for $1 \leq n \leq N$ (recall that $\omega_{1}=\omega_{-}$and $\omega_{N+1}=\omega_{+}$). The regularizing matrix $\mathbf{E}$ is real non negative diagonal and defined by $E_{m, m}=\epsilon_{m}$ for $1 \leq m \leq J$ and by $E_{m, m}=E_{K+m, K+m}=\epsilon_{m}^{\prime}$ for $J+1 \leq m \leq J+K$.

Solving this least-square problem with the constraint that $\boldsymbol{\mu}$ is real valued yields

$$
\boldsymbol{\mu}=\left[\operatorname{Re}\left(\mathbf{M}^{*} \mathbf{W}^{*} \mathbf{W} \mathbf{M}\right)+\boldsymbol{E}\right]^{-1} \operatorname{Re}\left(\mathbf{M}^{*} \mathbf{W}^{*} \mathbf{W H}\right) .
$$

This result is obtained by decomposing the complex values as $x+i y$, solving the problem and recomposing the result into the compact form (69).

\subsection{Results in the frequency domain}

The results of this optimization are presented in Fig. 9 for the following parameters: $\beta=0.3, \tau=1, \omega_{-}=10^{-4}, \omega_{+}=10^{5}, \omega_{n}=\omega_{-}\left(\omega_{+} / \omega_{-}\right)^{\frac{n-1}{N-1}}$ for $1 \leq n \leq N$ with $N=200$. For the cuts $\mathcal{C}_{h}$, we choose $\xi_{j}=10^{\frac{j-4}{2}}$ for $1 \leq j \leq J$ with $J=4$, $\xi_{k}^{\prime}=10^{\frac{k-1}{2}}$ and $\omega_{k}^{\prime}=\operatorname{Im}\left(s_{1}\right)=$ for $1 \leq k \leq K$ with $K=8$ (see Fig. 7 (1)a,b). For the cuts $\mathcal{C}_{c}$, we choose $\xi_{j}=10^{\frac{j-4}{4}}$ for $1 \leq j \leq J$ with $J=16, \xi_{k}^{\prime}=\operatorname{Re}\left(s_{1}\right)$ and 

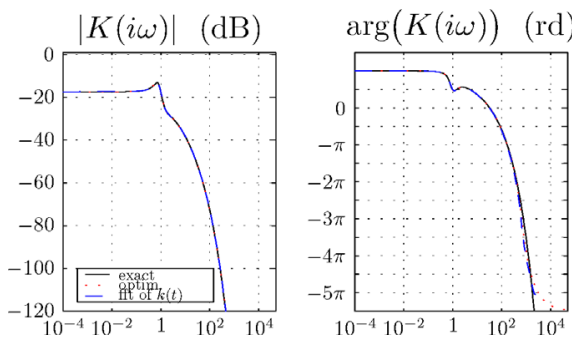

(a)
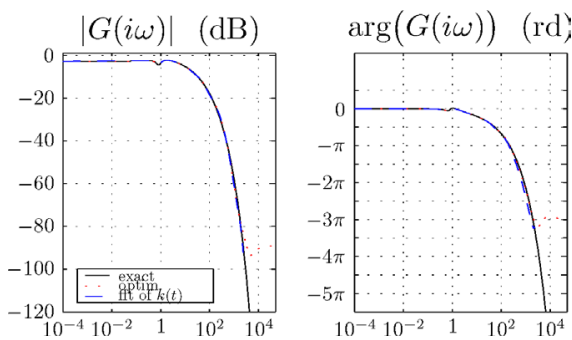

(d)
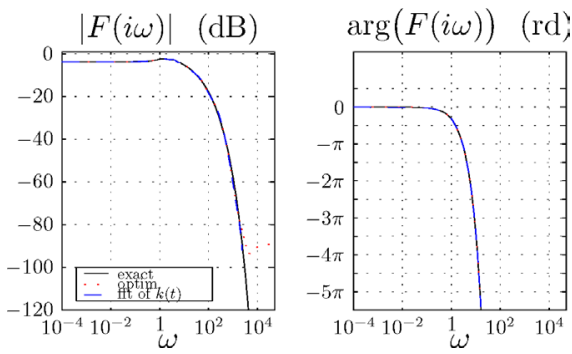

(g)

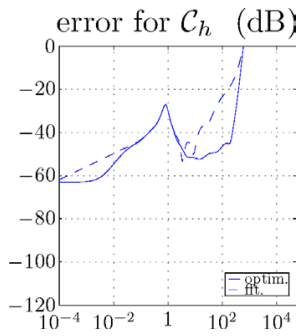

(b)

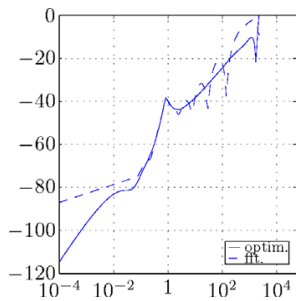

(e)

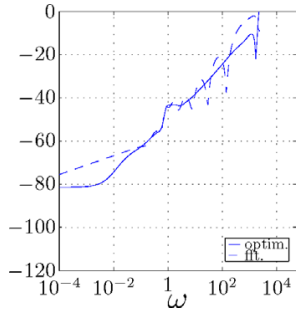

(h)

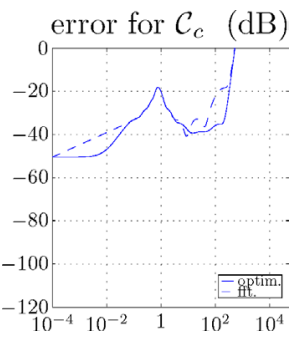

(c)

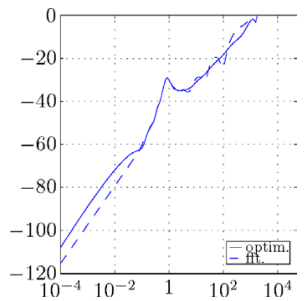

(f)

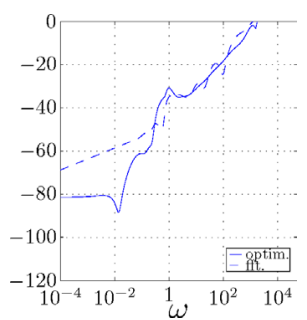

(i)

Fig. 9. Bode diagrams of the exact transfer functions (-), of approximated functions for the cuts $\mathcal{C}_{h}(:)$ and the Fourier transform of the corresponding discrete-time simulations (- $): K(i \omega)$ (a), $G(i \omega)(\mathrm{d}), H(i \omega)$ (g). The transfer functions $G$ are built from $\breve{G}$ thanks to Eq. (49), and $H$ thanks to $G, K$ and delays thanks to Eq. (18). Figures (b), (e), (h) represent the corresponding relative errors in decibels $20 \log _{10}|\tilde{H}(i \omega) / H(i \omega)-1|$ for the cuts $\mathcal{C}_{h}$ and (c), (f), (i), for the cuts $\mathcal{C}_{c}$.

$\omega_{k}^{\prime}=\frac{k}{K+1} \operatorname{Im}\left(s_{1}\right)$ for $1 \leq k \leq K$ with $K=2$ (see Fig. 7(1)c,d). For these parameters, no regularization is necessary so that we choose $\mathbf{E}=\mathbf{0}$. The corresponding approximations all yield systems of dimension $J+2 K=20$.

Note that the adimensional angular frequencies $\omega_{-}$and $\omega_{+}$(see Table 1 for the denormalization) allow to encompass nine decades (rather than 4 for the physical audio range). This allows one to show that the method yields satisfying results for various curvatures and lengths associated to a given set of adimensional parameters. 


\subsection{Time-domain simulation}

The finite-dimensional realizations of the approximated transfer functions are

$$
\begin{aligned}
\partial_{t} \phi_{j}(t)= & -\xi_{j} \phi_{j}(t)+u(t), \quad 1 \leq j \leq J, \\
\partial_{t} \phi_{k}^{\prime}(t)= & \left(-\xi_{k}^{\prime}+i \omega_{k}^{\prime}\right) \phi_{k}^{\prime}(t)+u(t), \quad 1 \leq k \leq K, \\
\tilde{y}(t)= & \sum_{j=1}^{J} \mu_{j} \phi_{j}(t)+2 \operatorname{Re} \sum_{k=1}^{K} \mu_{k}^{\prime} \phi_{k}^{\prime}(t), \\
z(t)= & \sum_{j=1}^{J}\left(-\xi_{j} \breve{\mu}_{j}\right) \phi_{j}(t)+2 \operatorname{Re} \sum_{k=1}^{K}\left(-\xi_{k}^{\prime}+i \omega_{k}^{\prime}\right) \breve{\mu}_{k}^{\prime} \phi_{k}(t) \\
& +\left[G(0)+\sum_{j=1}^{J} \breve{\mu}_{j}+2 \operatorname{Re} \sum_{k=1}^{K} \breve{\mu}_{k}^{\prime}\right] u(t) .
\end{aligned}
$$

Approximating $u(t)$ by its sample and hold version, that is $u(t) \approx u\left(t_{n}\right)$ for $t_{n} \leq$ $t<t_{n+1}$, Eqs. (70)-(73) become, in the discrete-time domain,

$$
\begin{aligned}
\phi_{j}\left(t_{n}\right)= & \alpha_{j} \phi_{j}\left(t_{n-1}\right)+\frac{\alpha_{j}-1}{-\xi_{j}} u\left(t_{n-1}\right), \\
\phi_{k}^{\prime}\left(t_{n}\right)= & \alpha_{k}^{\prime} \phi_{k}^{\prime}\left(t_{n-1}\right)+\frac{\alpha_{k}^{\prime}-1}{-\xi_{k}^{\prime}+i \omega_{k}^{\prime}} u\left(t_{n-1}\right), \\
\tilde{y}\left(t_{n}\right)= & \sum_{j=1}^{J} \mu_{j} \phi_{j}\left(t_{n}\right)+2 \operatorname{Re} \sum_{k=1}^{K} \mu_{k}^{\prime} \phi_{k}^{\prime}\left(t_{n}\right), \\
\tilde{z}\left(t_{n}\right)= & \sum_{j=1}^{J}\left(-\xi_{j} \breve{\mu}_{j}\right) \phi_{j}\left(t_{n}\right)+2 \operatorname{Re} \sum_{k=1}^{K}\left(-\xi_{k}^{\prime}+i \omega_{k}^{\prime}\right) \breve{\mu}_{k}^{\prime} \phi_{k}^{\prime}\left(t_{n}\right) \\
& +\left[G(0)+\sum_{j=1}^{J} \breve{\mu}_{j}+2 \operatorname{Re} \sum_{k=1}^{K} \mu_{k}^{\prime}\right] u\left(t_{n}\right),
\end{aligned}
$$

where $t_{n}=n T_{s}, T_{s}$ is the sampling period, $\alpha_{j}=\mathrm{e}^{-\xi_{j} T_{s}}$ and $\alpha_{k}^{\prime}=\mathrm{e}^{\left(-\xi_{k}^{\prime}+i \omega_{k}^{\prime}\right) T_{s}}$.

The impulse responses $k\left(t_{n}\right), g\left(t_{n}\right)$ and $h\left(t_{n}\right)$ are simulated thanks to these recursive equations and delay lines. They are represented in Fig. 10 for the sampling frequency $F_{s}=\frac{10^{4}}{4 \pi}$. The corresponding relative errors in the frequency domain are computed from the discrete Fourier tranform of the first $2^{16}$ computed points of the impulse response (see Fig. 9).

\section{Generalization: Input-Ouput Representation of a Whole Waveguide}

In this section, all the variables are original ones $(t, s, \Upsilon$, etc.) and not adimensional

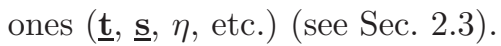



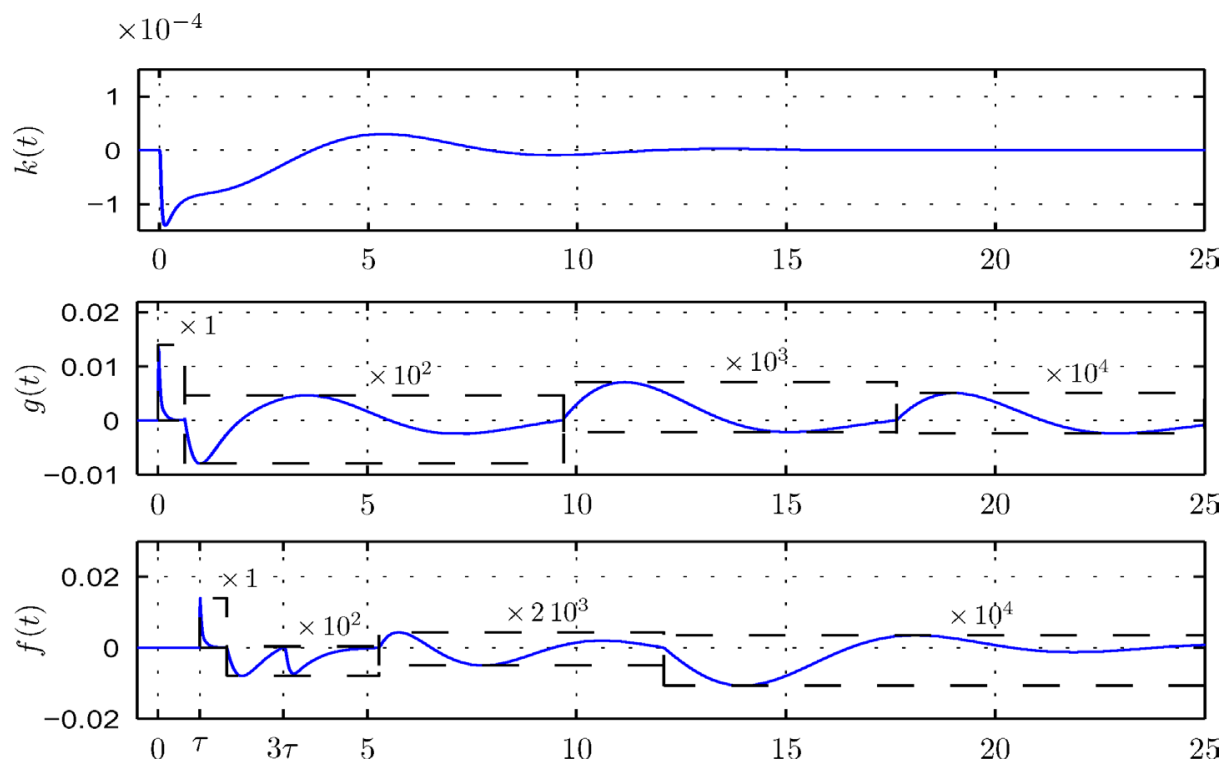

Fig. 10. Simulations in the (adimensional) time domain of the impulse responses of the subsystems $k(t)$ in (a), $g(t)$ in (b) and of the whole bell system $f(t)$ in (c). These impulse responses are obtained from the approximations and the cut $\mathcal{C}=\mathcal{C}_{h}$.

\subsection{Concatening quadripoles}

Under our 1D-modelling hypotheses, the $\ell$-continuity of $p, v$ and $\psi^{ \pm}$is satisfied if $^{19}$ the profile $\ell \mapsto r(\ell)$ has a continuous derivative. In such a case, a whole pipe can be represented by cascading the (denormalized) quadripoles $\mathbf{Q}_{n}(1 \leq n \leq N)$ where the pieces of pipes located at $\ell \in\left[\ell_{n-1}, \ell_{n}\right]\left(1 \leq n \leq N, \ell_{0}=0, \ell_{N}=L\right)$ are described by nearly constant coefficients $\varepsilon(\ell) \approx \varepsilon_{n}$ and $\Upsilon(\ell) \approx \Upsilon_{n}$ (see Fig. 11).

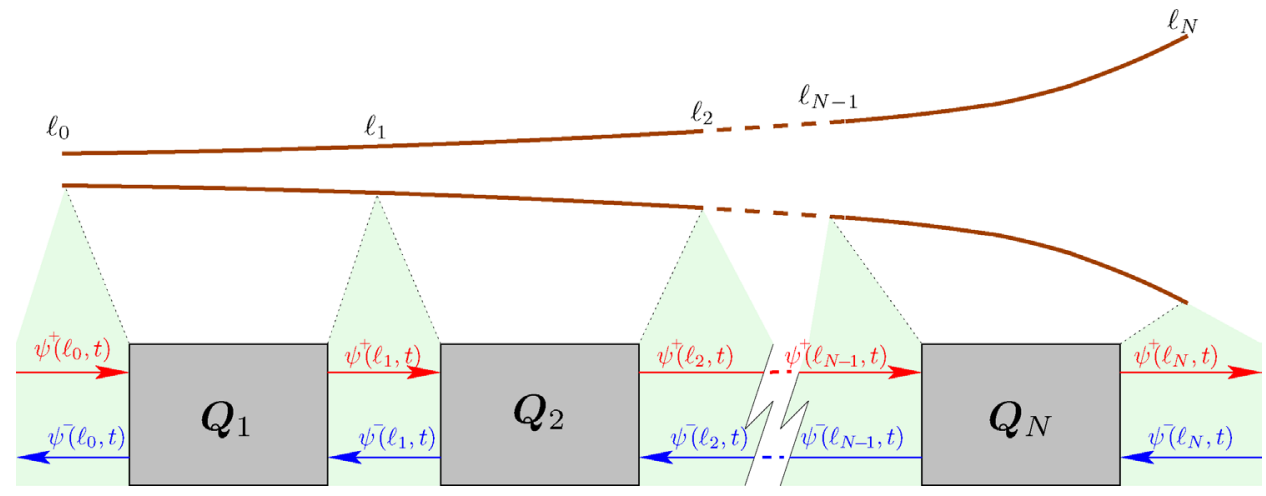

Fig. 11. Assuming that the profile $R$ is in $\mathcal{C}^{1}$, the whole pipe can be represented by concatenated quadripoles $\mathbf{Q}_{n}$. 
Standard boundary conditions on $(p, v)$ can be applied on the pipe by encapsulating the chain of $\mathbf{Q}_{n}$ with the conversion quadripoles given in Fig. 1. This defines a causal dynamical system of the whole acoustic system.

\subsection{Deriving a Kelly-Lochbaum structure}

Concatenating quadripoles $\mathbf{Q}_{n}(s)$ together or with the conversion quadripoles $C_{i}$ or $C_{0}$ creates instantaneous infinite loops (see the dashed arrow on Fig. 12(a)). This difficulty is overcome thanks to algebraic calculation: reformulating the equations of the junction $\left(y_{n+1}(s)=u_{n}(s)+E_{22}^{n}(s) y_{n}(s)\right.$ and $y_{n}(s)=u_{n+1}(s)+$ $\left.E_{11}^{n+1}(s) y_{n+1}(s)\right)$ so that $\left(y_{n}, y_{n+1}\right)$ are given as expressions of $\left(u_{n}, u_{n+1}\right)$ cancels this loop (see Fig. 12(b)). The stability condition for the "de-looping" is $\forall s \in \mathbb{C}_{0}^{+}$, $\Delta_{n}(s) \neq 0$ where

$$
\Delta_{n}(s)=1-E_{22}^{n}(s) E_{11}^{n+1}(s) .
$$

Applying this result to the concatenation of quadripoles $C_{i}, \mathbf{Q}_{n=1, \ldots, N}$ and $C_{0}$ yields the Kelly-Lochbaum structure presented in Fig. 13. The crucial point is that the loops of this structure include a delay, thus making the simulation possible.

The transfers of the quadripoles of junctions $\mathbf{J}_{n}(1 \leq n \leq N)$ are those of Fig. 12(b) with $E_{22}^{n}(s)=R_{n}(s), E_{11}^{n+1}(s)=R_{n+1}(s), \Delta_{n}(s)=1-R_{n}(s) R_{n+1}(s)$. The conversion quadripoles are of $\left\{E_{21}, E_{12}, E_{11}, E_{22}\right\}$ type. At the input, $\mathbf{C}_{i}$

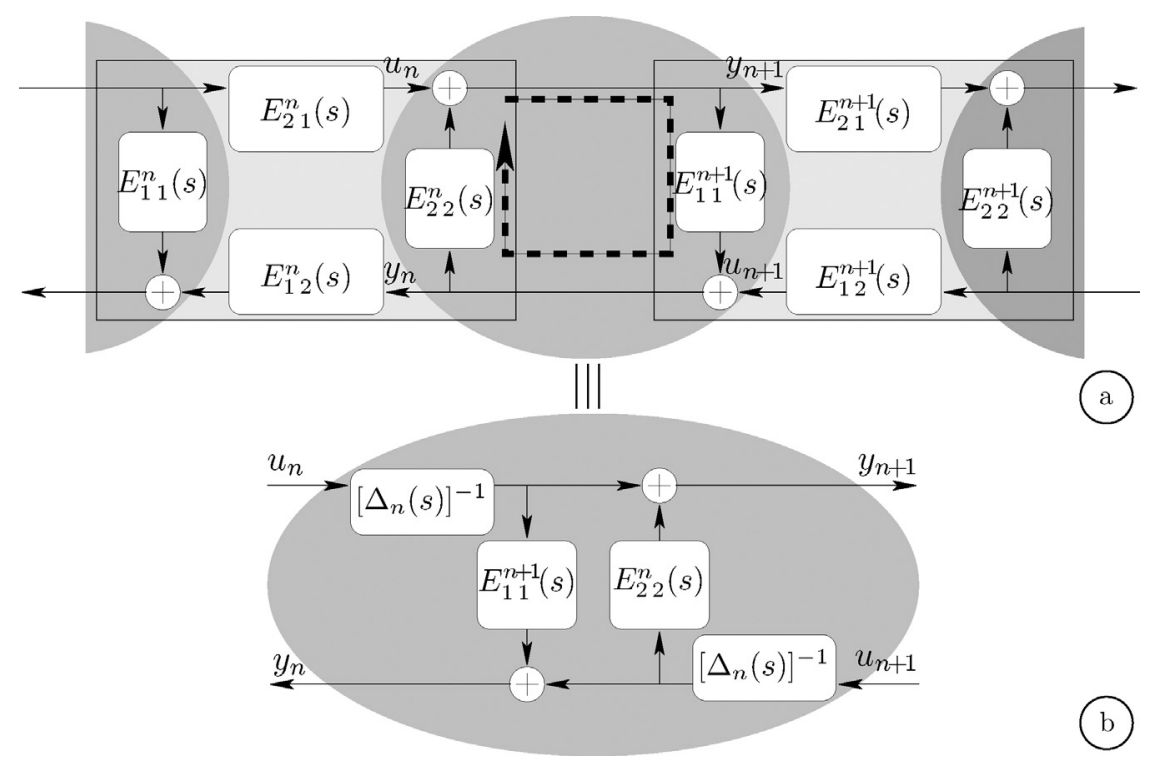

Fig. 12. Infinite loop (dashed arrow) created by connecting two quadripoles $\left\{E_{21}, E_{12}, E_{11}\right.$, $\left.E_{22}\right\}$ (a), and equivalent junction without loop $\left(\Delta_{n}(s)\right.$ is defined in Eq. (78)) (b). 


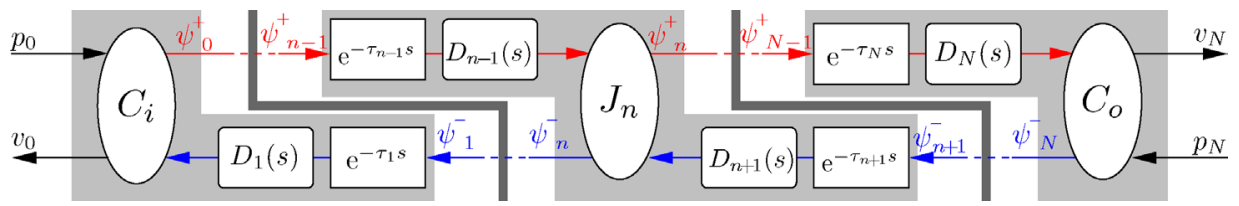

Fig. 13. Kelly-Lochbaum structure for a whole waveguide. Note that the denormalized delays are $\tau_{n}=\left(\ell_{n}-\ell_{n-1}\right) / c_{0}$ for $1 \leq n \leq N$.

corresponds to

$$
\begin{aligned}
& E_{21}^{C_{i}}(s)=r(0) / \Delta_{0}(s) \\
& E_{12}^{C_{i}}(s)=-2 /\left[\rho_{0} c_{0} r(0) \Delta_{0}(s)\right] \\
& E_{11}^{C_{i}}(s)=\left[1+\zeta_{0} / s-2 R_{1}(s) / \Delta_{0}(s)\right] /\left(\rho_{0} c_{0}\right), \\
& E_{22}^{C_{i}}(s)=-1 / \Delta_{0}(s)
\end{aligned}
$$

where $\Delta_{0}(s)=1+R_{1}(s)$ and $\zeta_{\ell}=r^{\prime}(\ell) / r(\ell)$. At the output, $\mathbf{C}_{0}$ corresponds to

$$
\begin{aligned}
& E_{21}^{C_{0}}(s)=2 /\left[\rho_{0} c_{0} r(L) \Delta_{N}(s)\right], \\
& E_{12}^{C_{0}}(s)=r(L) / \Delta_{N}(s), \\
& E_{11}^{C_{0}}(s)=-1 / \Delta_{N}(s), \\
& E_{22}^{C_{0}}(s)=\left[-1-\zeta_{L} / s+2 R_{N}(s) / \Delta_{N}(s)\right] /\left(\rho_{0} c_{0}\right),
\end{aligned}
$$

where $\Delta_{N}(s)=1+R_{N}(s)$.

Adapting the methods presented for the bell transfer functions in Secs. 4 and 5 to those of $\mathbf{C}_{i}, \mathbf{J}_{n}$ and $\mathbf{C}_{0}$ (see Sec. 7.1), the whole Kelly-Lochbaum structure of Fig. 13 will give rise to well-known and widely used digital filters ${ }^{20}$ where delays can be implemented, thanks to circular buffers.

\section{Perspectives and Open Questions}

\subsection{Perspectives}

\subsubsection{Transfer functions of concatenated quadripoles}

In the quadripole of junctions $\mathbf{J}_{n}$ (see Fig. 13), the irrational transfer functions have five branching points instead of three because of $\Delta_{n}(s)=1-R_{n}(s) R_{n+1}(s)$ (see Sec. 6.2). Namely, branching points are $s=0$ and two pairs $\left(s_{1}, \overline{s_{1}}\right)$, one pair being associated to one cell. Then, it could seem necessary to extend our technique to the case of five cuts. This can be avoided by choosing, for each quadripole $\mathbf{J}_{n}$, one cut which includes a connection between $s_{1}^{\mathbf{Q}_{n}}$ and $s_{1}^{\mathbf{Q}_{n+1}}$, the complex conjugate cut and $\mathbb{R}^{-}$. Thus, the realization in subsystems with delays of $R_{n}$ involved in $\mathbf{J}_{n}$ and that involved in $\mathbf{J}_{n-1}$ will be different. The cuts of these subsystems can be chosen independently since the only cut of $R_{n}$ is $\mathbb{R}^{-}$(see Sec. 2.4.2). 


\subsection{2. $\mathcal{C}$-contour techniques}

The $\mathcal{C}$-contour (or usually called $\Gamma$-contour ${ }^{21}$ ) is an alternative to the use of diffusive representations. It is deeply based on the Bromwich inversion formula of the Laplace transform, with a contour $\mathcal{C} \subset \mathbb{C}_{0}^{-}$which encircles all the singularities (poles, branching points, cuts, etc.). In this case, the time-domain representation is obtained, which involves as complex-valued weight $\mu(\gamma)=H(\gamma)$, namely:

$$
\begin{aligned}
\partial_{t} X_{\gamma}(t) & =\gamma X_{\gamma}(t)+u(t), \quad \gamma \in \mathcal{C}_{\gamma}, \\
y(t) & =\int_{\mathcal{C}_{\gamma}} H(\gamma) X_{\gamma}(t) \mathrm{d} \gamma .
\end{aligned}
$$

The well-posedness condition now reads $\int_{\mathcal{C}_{\gamma}}\left|\frac{\mu_{\gamma}}{1-\gamma}\right| \mathrm{d} \gamma<+\infty$ must be satisfied.

The diffusive representations can be interpreted as a limit case of a $\mathcal{C}$-contour which would embrace the cuts from both sides and eventually stick to them. This cut technique captures the information through the discontinuity of the transfer functions across the cuts (this is represented by $\mu$, see e.g. (26) or (31)).

The $\mathcal{C}_{\gamma}$-contour techniques express the same information on a somewhat averaged form: on one hand, it does not require to compute the jump on the discontinuity, but on the other hand, they seem to be less favorable to yield good approximations by systems of finite dimension with low orders.

\subsubsection{Optimizing pole placement on the cut}

An improvement of the optimization presented in Sec. 5.1.2 consists in minimizing the criterion (63) also with respect to the poles $\left\{-\xi_{j}\right\}$ and $\left\{\gamma_{k}\right\}$, which are required to remain on the cuts and preserve hermitian symmetry. This problem is now a nonlinear optimization problem under constraint. A straightforward future work will consists in applying classical method (Lagrange multipliers, Kuhn-Tucker optimality conditions, etc.).

\subsection{Open questions}

\subsubsection{Stability of a whole waveguide}

For sound synthesis, it is most important to ensure the stability of a whole waveguide made of concatenated quadripoles (see Fig. 11). In the simpler case of concatenation of cylinders, the waveguide is made of lossy transmissions (delays and damping operators) and real reflection coefficients; the stability of this system can then be proved as in Matignon. ${ }^{22}$ In our case, the reflections are no longer represented by real constant coefficients, but by complex-valued transfer functions, so that this proof cannot be used as such. Moreover, it is not known if an extension will be available: further investigation will be carried out.

\subsubsection{Negative curvatures}

When $\eta=-1$, Eq. (34) becomes $\sigma^{4}+2 \beta \sigma^{3}-1=0$. The roots of this equation give rise to three branching points in the $s$-plane other than $s=0$ : two complex 
conjugated $s_{1}$ and $s_{2}=\overline{s_{1}}$ with negative real-part, and $s_{3}$ which is a strictly positive real. The branching point $s_{3}$ accounts for instability of the subsystems associated to the transfer functions $E(s), D(s), G(s)$ and $K(s)$ (see (15)-(20)).

Nevertheless, 0 is the only branching point and $\mathbb{R}^{-}$the only cut of the transfer functions $T(s), R(s), F(s)$ (see Secs. 2.4.2-2.5) as well as of the quadripoles $\mathbf{C}_{i}(s)$, $\mathbf{J}_{n}(s)$ and $\mathbf{C}_{0}(s)$ (see Sec. 6.2). These are functions of $\Gamma(s)^{2}$. All the corresponding "macro-systems" are then stable. This is confirmed by the Webster equation: the case $\beta=0$ is known to be conservative and gives rise to trapped modes ${ }^{23}$ for negative curvatures $(\eta=-1)$.

A future work could consist in looking for a decomposition of these "macrosystems" into delays and "subsystems" (different from $E(s)$ and $D(s)$ ) which would involve the only cut $\mathbb{R}^{-}$. Finding such a realizable decomposition is not straightforward but will allow to simulate the propagation in any waveguide, including positive, zero and negative curvatures.

\section{Conclusion}

In this paper, we consider a one-dimensional model of propagation inside axisymmetric pipes which takes into account both varying cross-sections and visco-thermal losses at the wall. We give a new definition of travelling waves which extends that of plane waves for cylinders and circular waves for cones. This definition allows a straightforward formulation thanks to causal quadripoles: since they are causal systems, they are well-adapted to simulation in the time domain. Now, apart from delays, these quadripoles involve pseudo-differential operators in time, which do not have a state-space realization of finite dimension.

Our work consists in building up finite-dimensional approximations. This is performed in three steps. Firstly, a careful analysis of the poles and branching points of the transfer functions is carried out. Secondly, cuts linking the branching points are chosen, yielding equivalent infinite-dimensional representations of these transfer functions. Finally, choosing a finite number of poles on these cuts and optimizing the weights thanks to an audio criterion leads to accurate finite-dimensional approximations.

As the example of a baffled bell illustrates, two systems of order 20 and two delays can approximate the bell transfer function with a relative error less than $1 \%$ on more than six decades.

\section{Appendix A. Resolution of the Webster-Lokshin Model in the Laplace Domain}

The solution of (10)-(11) is given by

$$
\begin{aligned}
\boldsymbol{\Psi}(\ell, s) & =\exp (\boldsymbol{\Theta}(s) \ell) \boldsymbol{\Psi}(0, s), \\
\exp (\boldsymbol{\Theta}(s) \ell) & =\left[\begin{array}{ll}
E_{11}(\ell, s) & E_{12}(\ell, s) \\
E_{21}(\ell, s) & E_{22}(\ell, s)
\end{array}\right],
\end{aligned}
$$




$$
\begin{aligned}
E_{11}(\ell, s) & =\cosh (\tau \Gamma(s) \ell)-\frac{1}{2}\left(\frac{\Gamma(s)}{s}+\frac{s}{\Gamma(s)}\right) \sinh (\tau \Gamma(s) \ell), \\
E_{12}(\ell, s)=-E_{21}(\ell, s) & =-\frac{1}{2}\left(\frac{\Gamma(s)}{s}-\frac{s}{\Gamma(s)}\right) \sinh (\tau \Gamma(s) \ell), \\
E_{22}(\ell, s) & =\cosh (\tau \Gamma(s) \ell)+\frac{1}{2}\left(\frac{\Gamma(s)}{s}+\frac{s}{\Gamma(s)}\right) \sinh (\tau \Gamma(s) \ell),
\end{aligned}
$$

for $\ell \in[0,1], s \in \mathbb{C}_{0}^{+}$, where $\Gamma(s)^{2}=s^{2}+2 \beta s^{\frac{3}{2}}+\eta$ and $\boldsymbol{\Psi}(\ell, s)=$ $\left[\Psi^{+}(\ell, s), \Psi^{-}(\ell, s)\right]^{\mathrm{T}}$. Note that, the power series of $\exp (\boldsymbol{\Theta}(s) \ell)$ shows that it is a function of $\Gamma(s)^{2}$ only.

We choose to define the quadripole $\mathbf{Q}$ for the inputs $\left[\psi^{+}(\ell=0, t), \psi^{-}(\ell=1, t)\right]$ and the outputs $\left[\psi^{+}(\ell=1, t), \psi^{-}(\ell=0, t)\right]$ (see Fig. 2). This preserves the direction of propagation of $\psi^{ \pm}$and defines a causal system (see proofs in Appendix C). The scattering matrix $\mathbf{Q}(s)$ is

$$
\mathbf{Q}(s)=\left[\begin{array}{cc}
E_{11}(1, s) & E_{12}(1, s) \\
0 & 1
\end{array}\right] \quad\left[\begin{array}{cc}
1 & 0 \\
E_{21}(1, s) & E_{22}(1, s)
\end{array}\right]^{-1} .
$$

The result yields the symmetric matrix detailed in Sec. 2.4.2.

\section{Appendix B. Analyticity and Boundedness of Transfer Functions in the Complex Right Half-Plane}

All the results below are given for $s \in \mathbb{C}_{0}^{+}$and $\eta \in\{0,1\}$.

\section{B.1. Notations and parametrizations}

Let $\sigma=a(1+i \lambda)$ with $a>0$ and $-1<\lambda<1$ define a parametrization of $s=\sigma^{2}=a^{2}\left(1-\lambda^{2}+2 i \lambda\right) \in \mathbb{C}_{0}^{+}$. Denoting $A(s)=\operatorname{Re}\left(\Gamma(s)^{2}\right)$ and $B(s)=\operatorname{Im}\left(\Gamma(s)^{2}\right)$, trigonometric formula for half-angles lead to $\Gamma(s)=\left(\sqrt{A(s)+\sqrt{A(s)^{2}+B(s)^{2}}}+\right.$ $\left.i B(s) / \sqrt{A(s)+\sqrt{A(s)^{2}+B(s)^{2}}}\right) / \sqrt{2} \in \mathbb{C}_{0}^{+}$(which is well defined since " $A(s)<0$ and $B(s)=0$ " can never occur together). The functions $A(s)$ and $B(s)$ are polynomials in the variables $a$ and $\lambda$ with coefficients depending on $\beta$ and $\eta$. But for the sake of conciseness, expressions are written in Secs. B.2-B.3 as functions of $a$, $\lambda$ and $s$ (without ambiguity since $(a, \lambda)$ and $s$ are in bijection).

\section{B.2. Function $E(s)$ is analytic and such that $|E(s)|<1$}

The assertion $|E(s)|<1$ is logically equivalent to $0<\operatorname{Re}(\bar{s} \Gamma(s))$ since $\Gamma(s)+s$ never cancels in $\mathbb{C}_{0}^{+}$(see Sec. 4.1.3). Now, $\sqrt{2} \operatorname{Re}(\bar{s} \Gamma(s))=a^{2}(1-$ $\left.\lambda^{2}\right) \sqrt{A(s)+\sqrt{A(s)^{2}+B(s)^{2}}}+2 a^{2} \lambda B(s) / \sqrt{A(s)+\sqrt{A(s)^{2}+B(s)^{2}}}>0$ since 
$\lambda B(s)>0$ and both terms never cancel together. The boundedness of $E(s)$ and the analyticity of $\Gamma(s)$ prove, from (15), that $E(s)$ is analytic.

\section{B.3. Function $D(s)$ is analytic and such that $|D(s)|<1$}

The assertion $|D(s)|<1$ is logically equivalent to $\operatorname{Re}(\Gamma(s)-s)>0$ which writes $\sqrt{A(s)^{2}+B(s)^{2}}>2[\operatorname{Re}(s)]^{2}-A(s)$. Now, $A(s)^{2}+B(s)^{2}-\left(2[\operatorname{Re}(s)]^{2}-A(s)\right)^{2}=$ $24 \beta\left(1-\lambda^{2}\right)\left[\left(\lambda^{2}-1\right)^{2}+1\right] a^{7}+4 \beta \lambda^{2}\left(3-\lambda^{2}\right)^{2} a^{6}+4 \eta\left(1-\lambda^{2}\right)^{2}>0$ proves that

$\sqrt{A(s)^{2}+B(s)^{2}}>\left|2[\operatorname{Re}(s)]^{2}-A(s)\right| \geq 2[\operatorname{Re}(s)]^{2}-A(s)$. Finally, from (16), $D(s)$ is analytic.

\section{B.4. Other functions}

The above results prove that $T(s), R(s), K(s)$ and $G(s)$ are analytic, that $|K(s)|=$ $\left|E(s) D(s)^{2}\right|<1$, so that $F(s)$ is also analytic in $\mathbb{C}_{0}^{+}$.

\section{Appendix C. Causality}

\section{C.1. Hardy spaces and Paley-Wiener theorem}

Let $\mathcal{L}^{2}\left(\mathbb{R}^{+}\right)=\left\{h: \mathbb{R}^{+} \rightarrow \mathbb{C} \mid h\right.$ is measurable and $\left.\int_{\mathbb{R}^{+}}|h(t)|^{2} \mathrm{~d} t<\infty\right\}$ denote the space of causal functions with finite energy, and

$$
\begin{aligned}
\mathbb{H}^{m}\left(\mathbb{C}_{0}^{+}\right)= & \left\{H: \mathbb{C}_{0}^{+} \rightarrow \mathbb{C} \mid H\right. \text { is holomorphic and } \\
& \left.\|H\|_{\mathbb{H}^{m}}=\sup _{\zeta>0}\left[\frac{1}{2 \pi} \int_{\mathbb{R}}|H(\zeta+i \omega)|^{m} \mathrm{~d} \omega\right]^{1 / m}<\infty\right\}
\end{aligned}
$$

denote the Hardy spaces for $m>0$. The Hardy spaces define transfer functions $H$ of causal systems, that is, the associated convolution kernels $h$ are zero for $t<0$ (see e.g. Dupraz ${ }^{6}$ ). The Paley-Wiener theorem gives the particular and more precise following result: under the Laplace transform, $\mathcal{L}^{2}\left(\mathbb{R}^{+}\right)$is isomorphic to $\mathbb{H}^{2}\left(\mathbb{C}_{0}^{+}\right)$(see e.g. Curtain and Zwart ${ }^{24}$ (p. 645) or Partington ${ }^{25}$ ).

\section{C.2. Causality for $E(s)$ and $D(s)$}

The functions $E(s)$ and $D(s)$ are analytic in $\mathbb{C}_{0}^{+}$and admit the asymptotic expansions, for $|s| \rightarrow+\infty$ with $s \in \mathbb{C}_{0}^{+}$,

$$
\begin{aligned}
& E(s)=\frac{\beta}{2} \frac{1}{\sqrt{s}}+o\left(\frac{1}{\sqrt{s}}\right), \\
& D(s)=\mathrm{e}^{-\tau \beta \sqrt{s}}\left[\mathrm{e}^{-\tau \beta^{2} / 2}+o(1)\right],
\end{aligned}
$$

so that $E \in \mathbb{H}^{m}\left(\mathbb{C}_{0}^{+}\right), \forall m>2$ and $D \in \mathbb{H}^{m}\left(\mathbb{C}_{0}^{+}\right), \forall m>0$. 
Diffusive Representations of Flared Acoustic Pipes

Table 2. Causality of systems under study throughout the paper.

\begin{tabular}{llllc}
\hline $\begin{array}{l}\text { Transfer } \\
\text { function }\end{array}$ & $\begin{array}{l}\text { Is equivalent to } \\
(\text { for }|s| \rightarrow+\infty \\
\left.\text { with } s \in \mathbb{C}_{0}^{+}\right)\end{array}$ & $\begin{array}{l}\text { Belongs to } \\
\mathbb{H}^{m}(\mathbb{C})\end{array}$ & $\begin{array}{l}\text { Causal } \\
\text { system }\end{array}$ & $\begin{array}{c}\text { Kernel of } \\
\text { finite energy }\end{array}$ \\
\hline$T(s)$ & $D(s)$ & $\forall m>0$ & yes & yes \\
$R(s)$ & $E(s)$ & $\forall m>2$ & yes & no \\
$F(s)$ & $D(s) \mathrm{e}^{-\tau s}$ & $\forall m>0$ & and delayed from $\tau$ & yes \\
$G(s)$ & $D(s)$ & $\forall m>0$ & yes & yes \\
$K(s)$ & $-E(s) D(s)^{2}$ & $\forall m>0$ & yes & yes \\
\hline
\end{tabular}

\section{C.3. Causality for other transfer functions}

Similar proofs yield the results compiled in Table 2 .

\section{Appendix D. Examples of Diffusive Representations}

\section{D.1. Example 1: Finite number of poles}

The function $H_{1}(s)=\sum_{n=0}^{N} \frac{\mu_{n}}{s+\xi_{n}}, \operatorname{Re}\left(\xi_{n}\right)>0$ is analytic on $\mathbb{C}_{0}^{+}$and meromorphic on $\mathbb{C}_{0}^{-}$. For $t<0$, the residues theorem can be applied to $H_{1}(s) \mathrm{e}^{s t}$ integrated on the Bromwich contour defined in Fig. 14(a) with $\varepsilon>1 / \max \left\{\operatorname{Re}\left(\xi_{n}\right)\right\}$. As no poles lie inside this contour, the sum of the residues is zero. The Jordan's lemma proves that the integral on the demi-circle reaches 0 for $\epsilon \rightarrow 0^{-}$and $\lambda \rightarrow+\infty$, since $t<0$ and $H_{1}(s)$ is analytic on $\mathbb{C}_{0}^{+}$. The integral on the contour in $\mathbb{C}_{0}^{-}$, divided by $2 i \pi$, gives the inverse Laplace transform, i.e. the impulse response $h_{1}(t)$ of the system. This implies that $h_{1}(t)=0$, proving the causality of the system.

For $t>0$, applying the same method with the contour defined in Fig. 14(b) with $\varepsilon>0, \lambda<0$. In this case, the Jordan's lemma can be used for the semi-circle in $\mathbb{C}_{0}^{-}$, and the contour encircles every poles for $\varepsilon \rightarrow 0^{+}$and $\lambda \rightarrow-\infty$ so that the

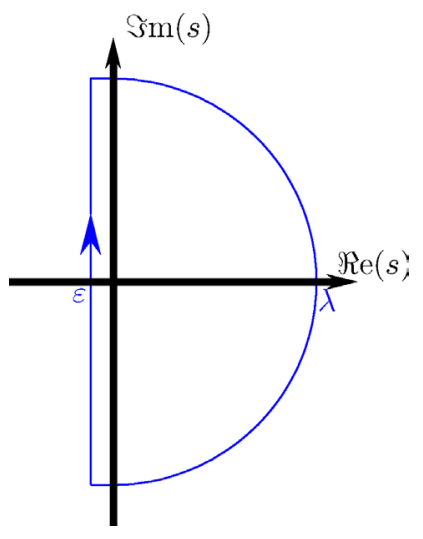

(a)

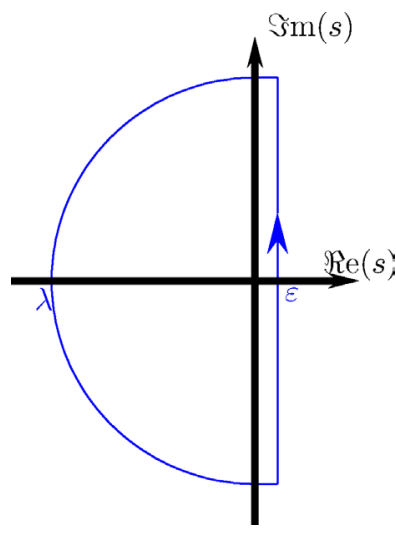

(b)

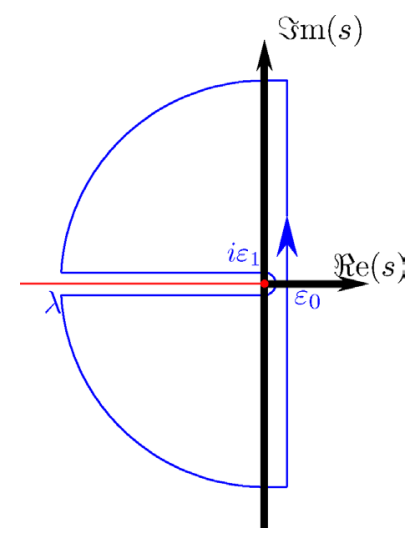

(c)

Fig. 14. Bromwich contours used for meromorphic functions on $\mathbb{C}$ which are analytic on $\mathbb{C}_{0}^{+}$. 
sum of residues is $\sum_{n=0}^{N} \mu_{n} \mathrm{e}^{-\xi_{n} t}$. The integral on the contour in $\mathbb{C}_{0}^{+}$, divided by $2 i \pi$, gives the inverse Laplace transform, so that finally,

$$
h_{1}(t)=\sum_{n=0}^{N} \mu_{n} \mathrm{e}^{-\xi_{n} t} \mathbf{1}_{t>0}(t) .
$$

\section{D.2. Example 2: Infinitely many poles}

The transfer $H_{2}(s)=\frac{\tanh (\sqrt{s})}{\sqrt{s}}$ seems to bring a new difficulty since the square root of a complex is defined except for a cut, generally taken as $\mathbb{R}^{-}$. This would prevent from considering contour Fig. 14(b) since it would systematically cross the discontinuity located in $s=\lambda \in \mathbb{R}^{-}$. But, the series expansion of $s \mapsto \tanh (s) / s$ makes only powers of $s^{2}$ appear and has an infinite radius of convergence, so that $H_{2}(s)$ has no cut on $\mathbb{C}$. The expansion ${ }^{26} \tanh (s) / s=2 \sum_{n=0}^{+\infty}\left[s^{2}+(n+1 / 2)^{2} \pi^{2}\right]^{-1}(s \in \mathbb{C})$ shows that $H_{2}(s)$ has only an infinite countable number of poles on $\mathbb{R}^{-}$. Using the dominated convergence theorem to swap the sum and the integral operator, the same method and contours as for example 1 yield the impulse response

$$
h_{2}(t)=2 \sum_{n=0}^{+\infty} \mathrm{e}^{-\left(n+\frac{1}{2}\right)^{2} \pi^{2} t} \mathbf{1}_{t>0}(t) .
$$

\section{D.3. Examples 3 and 4: A continuous line of singularities}

The transfer $H_{3}(s)=\frac{\mathrm{e}^{-\sqrt{s}}}{\sqrt{s}}$ has no poles but it is defined on $\mathbb{C}$ with the same cut as that of $s \mapsto \sqrt{s}$. For causal and stable transfers (singularities in $\mathbb{C}_{0}^{-}$) which maps real-valued inputs-outputs (hermitian symmetry in the convergence domain), the most natural analytic extension of $H_{3}(s)$ over $\mathbb{C}$ is obtained choosing the cut $\mathbb{R}^{-}$. Note that choosing the cut in $\mathbb{C}_{0}^{-}$has no influence on the impulse response of the associated causal system since it does not mofify $H_{3}(s)$ in its convergence domain.

Applying the residue theorem with the contour Fig. 14 (c) for $\varepsilon_{0} \rightarrow 0^{+}, \varepsilon_{1} \rightarrow 0^{+}$ and $\lambda \rightarrow-\infty$, leads to ${ }^{13}$

$$
h_{3}(t)=\int_{0}^{+\infty} \mu_{3}(\xi) \mathrm{e}^{-\xi t} \mathrm{~d} \xi=\frac{\mathrm{e}^{-\frac{1}{4 t}}}{\sqrt{\pi t}}, \quad \forall t>0,
$$

where $^{4}$

$$
\mu_{3}(\xi)=\frac{1}{2 i \pi}\left[H\left(-\xi+i 0^{-}\right)-H\left(-\xi+i 0^{+}\right)\right]=\frac{\cos (\tau \sqrt{\xi})}{\pi \sqrt{\xi}} .
$$

This is still possible according to the dominated convergence theorem since

$$
\int_{0}^{+\infty} \frac{\left|\mu_{3}(\xi)\right|}{1+\xi} \mathrm{d} \xi<+\infty
$$

Another example is the fractional integrator defined by the transfer $H_{4}(s)=s^{-a}$ with a cut on $\mathbb{R}^{-}$for $0<a<1$, and which is associated to the diffusive symbol $\mu_{4}(\xi)=\frac{\sin (a \pi)}{\pi} \xi^{-a}$. 


\section{Appendix E. Weights of the Diffusive Representations of the Bell Transfer Functions}

The expression of the weights used for the bell are shown in Table 3. The two first lines precise the piece of the cut for which the weights are computed: $\mu_{\alpha, \mathcal{C}}$ denotes the weight associated to $K(s)$ computed on the part of the cut $\mathcal{C}$ which starts from the branching point $\alpha ; \breve{\mu}_{\alpha, \mathcal{C}}$ is associated to $\breve{G}(s)$. The two following lines detail the expressions from the compact expressions defined just below and where $\chi$ is defined in (36).

Table 3. Analytic expressions of the weigths $\mu$ involved in the bell transfer functions.

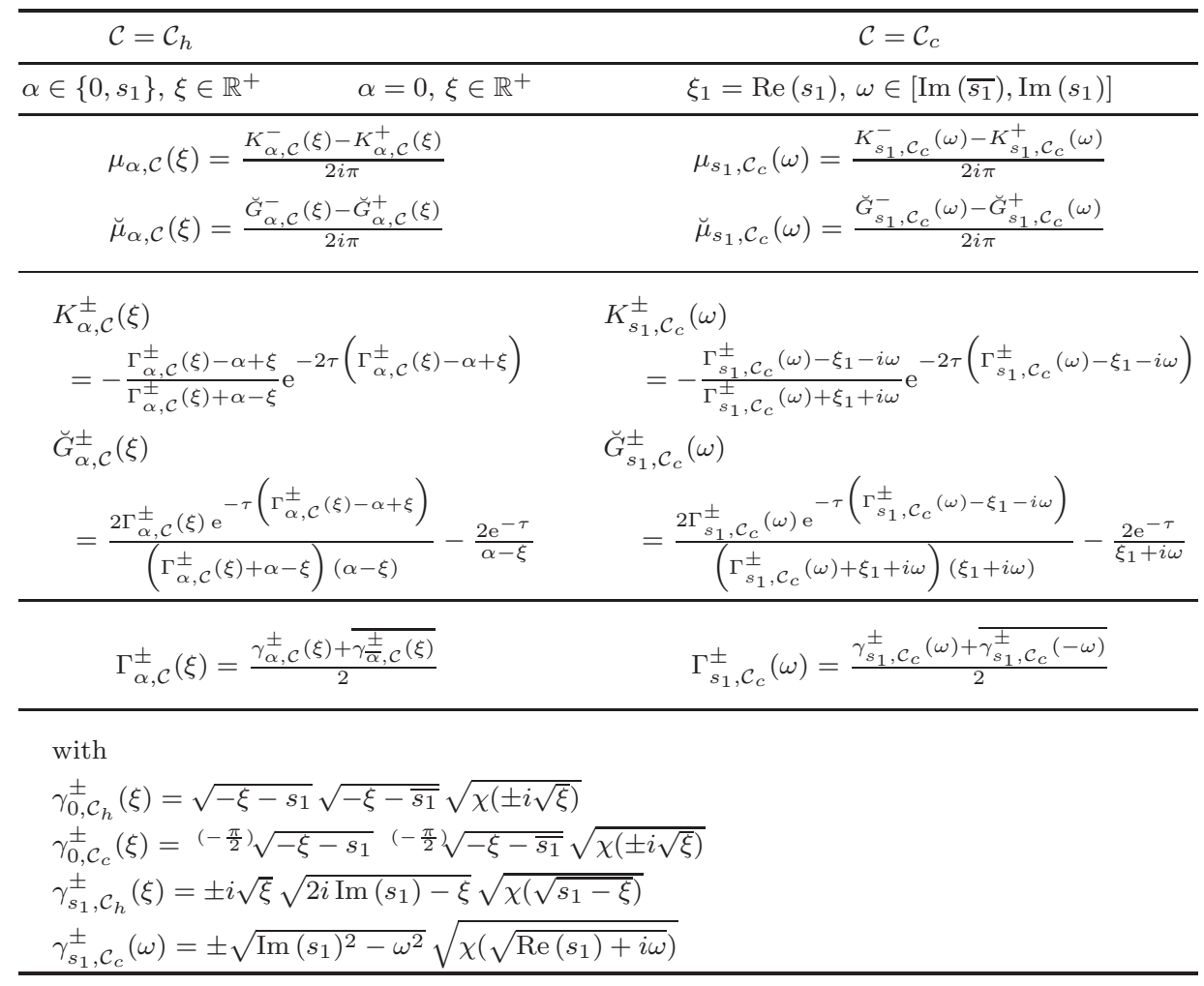

\section{References}

1. H. Haddar, T. Hélie and D. Matignon, A Webster-Lokshin model for waves with viscothermal losses and impedance boundary conditions: Strong solutions, in 6th Int. Conf. on Mathematical and Numerical Aspects of Wave Propagation Phenomena, Jyväskylä, Finland, 2003 (INRIA, 2003), pp. 66-71.

2. H. Haddar and D. Matignon, Theoretical and numerical analysis of the WebsterLokshin model, Technical report, INRIA, 2005.

3. T. Hélie, Unidimensional models of acoustic propagation in axisymmetric waveguides, J. Acoust. Soc. Amer. 114 (2003) 2633-2647. 
4. D. Matignon, Stability properties for generalized fractional differential systems, ESAIM Proc. 5 (1998) 145-158.

5. M. Bruneau, Manuel d'acoustique Fondamentale (Hermès, 1998).

6. J. Dupraz, La Théorie des Distributions et ses Applications (Cépadues, 1977).

7. D. Matignon and B. d'Andréa-Novel, Spectral and time-domain consequences of an integro-differential perturbation of the wave pde, in 3rd Int. Conf. on Math. and Numer. Aspects of Wave Propagation Phenomena, Mandelieu, France, April 1995 (SIAM, 1995), pp. 769-771.

8. M. Taylor, Pseudo-Differential Operators, Princeton Mathematical Series, Vol. 34 (Princeton Univ. Press, 1981).

9. O. J. Staffans, Well-posedness and stabilizability of a viscoelastic equation in energy space, Trans. Amer. Math. Soc. 345 (1994) 527-575.

10. G. Montseny, Diffusive representation of pseudo-differential time-operators, ESAIM Proc. 5 (1998) 159-175.

11. D. Matignon and H. J. Zwart, Standard diffusive systems as well-posed linear systems, Syst. Control Lett., submitted.

12. D. Salamon, Infinite dimensional linear systems with unbounded control and observation: A functional analytic approach, Trans. Amer. Math. Soc. 300 (1987) 383-431.

13. M. Abramowitz and I. A. Stegun, Handbook of Mathematical Functions (Dover, 1970).

14. H. Zwart, Transfer functions for infinite-dimensional systems, Syst. Control Lett. 52 (2004) 247-255.

15. D. G. Duffy, Transform Methods for Solving Partial Differential Equations (CRC Press, 1994).

16. D. Héleschewitz, Analyse et simulation de systèmes différentiels fractionnaires et pseudo-différentiels linéaires sous représentation diffusive, Ph.D. thesis, Ecole Nationale Supérieure des Télécommunications, 2000.

17. T. Hélie and D. Matignon, Representations with poles and cuts for the time-domain simulation of fractional systems and in rational transfer functions, in Signal Processing (Elsevier, 2006).

18. E. Zwicker and R. Feldtkeller, Psychoacoustique-L'oreille, Récepteur D'information, Collection Technique et Scientifique des Télécommunications (Masson, 1981).

19. T. Hélie, Physical modeling of musical instruments with dynamic systems and inversion processes, Ph.D. thesis, Université Paris-Sud, Orsay, 2002.

20. J. O. Smith, Physical modeling synthesis update, Comput. Music J. 20 (1996) 44-56.

21. M. Dunau, Représentations diffusives de seconde espèce: Introduction et expérimentation, Master's thesis, DEA d'Automatique, Toulouse, 2000.

22. D. Matignon, Représentations en variables d'état de guides d'ondes avec dérivation fractionnaire, Ph.D. thesis, Université Paris-Sud, 1994, Appendix C.

23. D. P. Berners, Acoustics and signal processing techniques for physical modeling of brass instruments, Ph.D. thesis, Stanford University, 1999.

24. R. Curtain and H. Zwart, An Introduction to Infinite-Dimensional Linear Systems Theory (Springer-Verlag, 1995).

25. J. R. Partington, Linear Operators and Linear Systems, London Mathematical Society Student Texts, Vol. 60 (Cambridge Univ. Press, 2004).

26. H. Cartan, Elementary Theory of Analytic Functions of One or Several Complex Variables, Coll. Enseignement des Sciences (Hermann, 1961). 